\title{
Nucleosynthesis imprints from different Type la supernova explosion scenarios and implications for galactic chemical evolution
}

\author{
F. Lach ${ }^{1,2}$, F. K. Röpke ${ }^{1,3}$, I. R. Seitenzahl ${ }^{4}$, B. Coté ${ }^{5,6,7}$, S. Gronow ${ }^{1,2}$, and A. J. Ruiter ${ }^{4}$ \\ ${ }^{1}$ Heidelberger Institut für Theoretische Studien, Schloss-Wolfsbrunnenweg 35, 69118 Heidelberg, Germany \\ e-mail: florian. lachah-its.org \\ 2 Zentrum für Astronomie der Universität Heidelberg, Astronomisches Rechen-Institut, Mönchhofstr. 12-14, 69120 Heidelberg, \\ Germany \\ 3 Zentrum für Astronomie der Universität Heidelberg, Institut für Theoretische Astrophysik, Philosophenweg 12, 69120 Heidelberg, \\ Germany \\ ${ }^{4}$ School of Science, University of New South Wales, Australian Defence Force Academy, Canberra, ACT 2600, Australia \\ 5 Konkoly Observatory, Research Centre for Astronomy and Earth Sciences, MTA Centre for Excellence, Konkoly Thege Miklos \\ 15-17, 1121 Budapest, Hungary \\ 6 ELTE Eötvös Lorénd University, Institute of Physics, Pázmány Péter Sétány 1/A, Budapest 1117, Hungary \\ 7 National Superconducting Cyclotron Laboratory, Michigan State University, East Lansing, MI 48824, USA
}

Received 23 June 2020 / Accepted 19 October 2020

\begin{abstract}
We analyze the nucleosynthesis yields of various Type Ia supernova explosion simulations including pure detonations in subChandrasekhar mass white dwarfs; double detonations and pure helium detonations of sub-Chandrasekhar mass white dwarfs with an accreted helium envelope; a violent merger model of two white dwarfs; and deflagrations and delayed detonations in Chandrasekhar mass white dwarfs. We focus on the iron peak elements $\mathrm{Mn}, \mathrm{Zn}$, and $\mathrm{Cu}$. To this end, we also briefly review the different burning regimes and production sites of these elements, as well as the results of abundance measurements and several galactic chemical evolution studies. We find that super-solar values of $[\mathrm{Mn} / \mathrm{Fe}]$ are not restricted to Chandrasekhar mass explosion models. Scenarios including a helium detonation can significantly contribute to the production of $\mathrm{Mn}$, in particular the models proposed for calcium-rich transients. Although Type Ia supernovae are often not accounted for as production sites of $\mathrm{Zn}$ and $\mathrm{Cu}$, our models involving helium shell detonations can produce these elements in super-solar ratios relative to Fe. Our results suggest a re-consideration of Type Ia supernova yields in galactic chemical evolution models. A detailed comparison with observations can provide new insight into the progenitor and explosion channels of these events.
\end{abstract}

Key words. nuclear reactions, nucleosynthesis, abundances - methods: numerical - stars: abundances - supernovae: general white dwarfs - Galaxy: abundances

\section{Introduction}

Over the past two decades Type Ia supernovae (SNe Ia) have been the focus of interest of astrophysical studies primarily because of their application as distance indicators (Riess et al. 1998; Perlmutter et al. 1999) via the Phillips relation (Phillips 1993). As one of the signifcant sources for heavy elements in the Universe, however, they are also important as a main contributor to cosmic nucleosynthesis (Matteucci \& Greggio 1986; Matteucci \& Recchi 2001; Matteucci et al. 2006; Kobayashi et al. 2006, 2015, 2020; Kobayashi \& Nomoto 2009). Although SNe Ia have been intensely studied in observational and theoretical approaches, the questions concerning their progenitors and explosion mechanisms remain open.

There is broad agreement that SNe Ia originate from the thermonuclear explosions of carbon-oxygen white dwarf (WD) stars (Hoyle \& Fowler 1960). In some cases, however, also oxygenneon WDs (Marquardt et al. 2015) or hybrid carbon-oxygenneon WDs (Kromer et al. 2015; Willcox et al. 2016) may give rise to similar events. In most scenarios the explosion is triggered by the interaction with a binary companion. This rather unsharp characterization leaves room for a "zoo" of possible progenitor (e.g., Wang \& Han 2012) and explosion scenarios (see, e.g., Hillebrandt \& Niemeyer 2000; Hillebrandt et al. 2013). Potential progenitors can be subdivided into the singledegenerate (SD) scenario involving one WD accompanied by a main sequence, giant, or helium star (Whelan \& Iben 1973) and the double-degenerate (DD) scenario consisting of a binary system of two WDs (Iben \& Tutukov 1984). Another possibility, the core-degenerate scenario, has been proposed by Kashi \& Soker (2011). Here a WD merges with a post-asymptotic giant branch (AGB) core already during the common envelope phase, and forms a new WD above the Chandrasekhar mass $\left(M_{\mathrm{Ch}}\right)$ that is stabilized by rotation. As the rotation slows down it might explode as a SN Ia.

From the explosion modeling point of view and the implied nucleosynthesis output, however, the mass of the WD at the time of explosion is the fundamental parameter (Seitenzahl et al. 2017). Generally, near- $M_{\mathrm{Ch}}$ models are distinguished from models in which the exploding WDs are significantly below the Chandrasekhar mass limit of approximately $1.4 M_{\odot}$. Finally, the characteristics of the explosion is governed by the combustion 
mechanism. A thermonuclear combustion wave is formed via a runaway process during convective burning, due to dynamical interaction in a WD merger, or by converging shock waves. In the first case a subsonic deflagration propagates via heat conduction. The two other cases may lead to the formation of a supersonic detonation where, in contrast, the fuel is heated and burned by the compression of a shock wave (see, e.g., Röpke et al. 2017 for a review of thermonuclear combustion in SNe Ia).

It is not clear yet which of the various possible explosion mechanisms can account for SNe Ia. In addition to the bulk of normal SNe Ia obeying the Phillips relation, a variety of subclasses of SNe Ia have been identified (see Taubenberger 2017 for a review), and therefore it is plausible that more than one scenario contributes to the overall class of SNe Ia.

One approach to check the validity of a certain scenario is to conduct multidimensional hydrodynamical simulations of the explosion phase together with the subsequent calculation of synthetic observables, such as light curves and spectra. These can then be compared to observations of SNe Ia and the initial model can be accepted, discarded, or adjusted accordingly. This exercise has been carried out during the past years for a variety of explosion scenarios, and although suitable explanations of subclasses could be identified, there is no fully convincing model for the bulk of normal SNe Ia yet (Blinnikov et al. 2006; Kasen \& Plewa 2007; Blondin et al. 2011; Röpke et al. 2012; Seitenzahl et al. 2013a; Sim et al. 2013; Fink et al. 2014; Kromer et al. 2013; Townsley et al. 2019; Gronow et al. 2020).

Another test of the realization of specific explosion scenarios are abundance measurements in stars combined with galactic chemical evolution (GCE) models (Matteucci et al. 2006; Travaglio et al. 2014; Kobayashi et al. 2011). Among other ingredients, these models assume certain rates, delay-time distributions, and nucleosynthesis yields for various kinds of $\mathrm{SNe}$ Ia and core-collapse supernovae (CCSNe). The enrichment of the investigated stellar population or galaxy with metals is then compared to stellar abundances derived from spectroscopy and thus allows us to infer the origin of a particular element or group of elements. This is the reason why the characteristic imprints of a certain explosion scenario are of great interest.

A prominent example is the case of the element manganese. It is widely accepted that the primary contribution to $\mathrm{Mn}$ stems from $\mathrm{SNe}$ Ia since the observed values of $[\mathrm{Mn} / \mathrm{Fe}]$ in the Galaxy increase from $[\mathrm{Fe} / \mathrm{H}] \approx-1$ to the solar value, and CCSN yields predict sub-solar values for [Mn/Fe] (Timmes et al. 1995; McWilliam 1997; Kobayashi et al. 2006, 2011, 2020; Kobayashi \& Nomoto 2009; Weinberg et al. 2019). This "SN Ia knee" has already been explained by Tinsley (1979), Greggio \& Renzini (1983), and Matteucci \& Greggio (1986) since it coincides nicely with the decrease in the $\alpha$-elements (i.e., $[\alpha / \mathrm{Fe}])$ produced by $\mathrm{CCSNe}$ from their super-solar plateau at lower $[\mathrm{Fe} / \mathrm{H}]$. Seitenzahl et al. (2013b) picked this up arguing that $\mathrm{Mn}$ is produced at high burning densities in normal freezeout from nuclear statistical equilibrium (NSE; see Sect. 2), and therefore it predominantly originates from $M_{\mathrm{Ch}}$ explosions. The best agreement with the data is achieved if SNe Ia arise equally from sub- $M_{\mathrm{Ch}}$ and $M_{\mathrm{Ch}}$ progenitors. These results have been further refined in the recent work of Eitner et al. (2020). Their non-LTE measurements of $\mathrm{Mn}$ in a sample of 42 stars in the Galaxy show a rather flat evolution of $[\mathrm{Mn} / \mathrm{Fe}]$ near the solar value lowering the contribution of $M_{\mathrm{Ch}} \mathrm{SNe}$ Ia to about $25 \%$. The trend in $[\mathrm{Mn} / \mathrm{Fe}]$ is not so clear in dwarf spheroidal galaxies (dSphs), but various works also find that a SN Ia contribution to $\mathrm{Mn}$ is required to explain observations (Cescutti et al. 2008; North et al. 2012; Cescutti \& Kobayashi 2017). Another study carried out by McWilliam et al. (2018) claims that the most metal-rich star in Ursa Minor, COS 171, was enriched by a low-metallicity, low-mass sub- $M_{\mathrm{Ch}}$ detonation. In particular, its low $[\mathrm{Mn} / \mathrm{Fe}]$ and $[\mathrm{Ni} / \mathrm{Fe}]$ values exclude a $M_{\mathrm{Ch}}$ origin. Furthermore, the sub-solar amounts of $\mathrm{Cu}$ and $\mathrm{Zn}$ in $\mathrm{COS} 171$ are also a hint for a low-mass progentior of the SN explosion which has enriched the star since these elements are produced in strong $\alpha$-rich freeze-out. Moreover, de los Reyes et al. (2020) attribute different $[\mathrm{Mn} / \mathrm{Fe}]$ values in $\mathrm{dSphs}$ to their specific star formation history. The combination of sub-solar $[\mathrm{Mn} / \mathrm{Fe}]$ and a short star burst, as seen in Sculptor, indicates a dominant role of sub- $M_{\mathrm{Ch}}$ explosions. In contrast, Fornax and Leo I show a long-lasting star formation and $[\mathrm{Mn} / \mathrm{Fe}]$ around the solar value, which points to an increasing enrichment via near- $M_{\mathrm{Ch}} \mathrm{SNe}$ Ia.

Our work analyzes the nucleosynthetic yields of various models for SNe Ia, namely pure deflagrations and delayed detonations in $M_{\mathrm{Ch}}$ WDs, as well as pure detonations and double detonations of sub- $M_{\mathrm{Ch}}$ WDs. The aim is not to go into detail about the observational implications of specific isotopes or to evaluate whether the model matches any observed SN Ia, but to identify specific abundance patterns that are characteristic for particular explosion scenarios, and therefore to constrain whether this scenario is required to produce the observed elemental and isotopic abundances. The key discriminant is the initial mass of the WD that sets its central density, mainly determining the freeze-out regime reached by the burning region. Normal freeze-out from NSE, for instance, requires high densities only reached in $M_{\mathrm{Ch}}$ WDs, and the presence of a helium detonation yields unique abundance patterns not produced in explosive carbon-oxygen burning. Moreover, electron capture significantly reduces the electron fraction at high densities, and therefore shifts the nucleosynthesis yields in NSE to more neutron rich isotopes (see Sect. 2.2). Thus, the occurrence of iron group elements (IGEs) with a considerable neutron excess suggests the Chandrasekhar mass scenario (Yamaguchi et al. 2015).

This work is structured in the following way. In Sect. 2 we first review the different burning regimes and then summarize the explosion models investigated. Section 3 presents the results of our nucleosynthesis analysis, and we discuss the implications of these results for the nature of the progenitor for SNe Ia. The elements $\mathrm{Mn}, \mathrm{Zn}$, and $\mathrm{Cu}$ are of particular interest. Because we find substantial amounts of the unstable radionuclides ${ }^{68} \mathrm{Ge},{ }^{68} \mathrm{Ga}$, and ${ }^{65} \mathrm{Zn}$, we briefly test their potential impact on the observables. In Sect. 4 we summarize our findings.

\section{Explosion models and burning regimes}

\subsection{Explosive silicon burning}

In a thermonuclear supernova explosion the innermost (i.e., densest) part of the WD star reaches temperatures sufficiently high for silicon burning, and thus significantly contributes to the production of IGEs. Woosley et al. (1973) divide the parameter space of this burning regime into three regions: the regime of incomplete silicon burning, alpha-rich freeze-out, and normal freeze-out from NSE (see Fig. 1). In NSE all the abundances from protons, neutrons, and $\alpha$-particles up to the iron peak have reached an equilibrium (i.e., the forward and reverse reactions cancel each other). In addition to the peak temperature $T_{\text {peak }}$ and density $\rho_{\text {peak }}$, the timescale on which a particular fluid element cools after the crossing of the burning front also determines the nucleosynthesis yields. Adiabatic cooling can be written as 


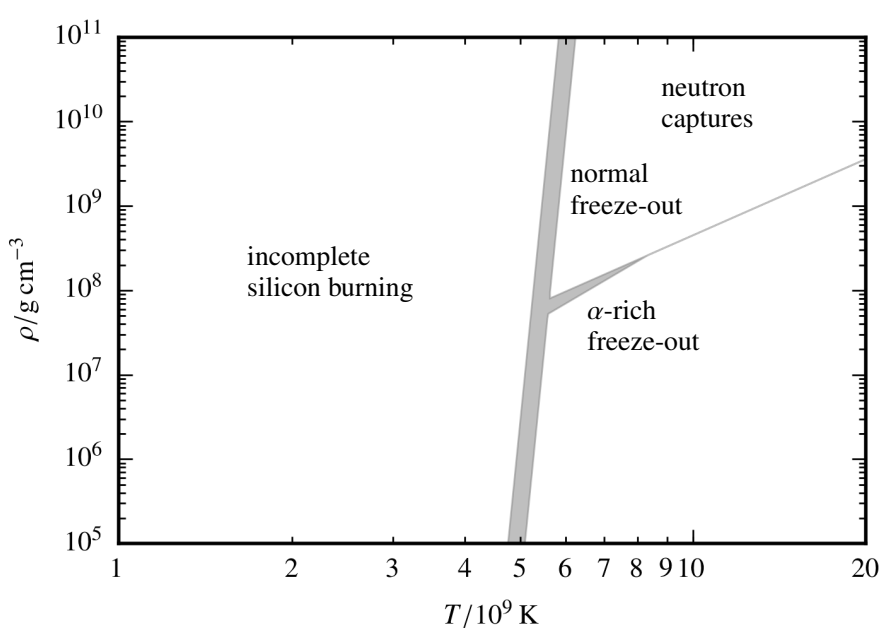

Fig. 1. Burning regimes in the $T-\rho$ plane according to Woosley et al (1973). Below a certain temperature the burning of silicon is incomplete. At high temperatures the state of nuclear statistical equilibrium is achieved and silicon is transformed into iron peak elements. This region is subdivided in the regime of normal freeze-out and $\alpha$-rich freeze-out The shaded area covers values of $\chi$ between 1 and 10 according to Eqs. (3) and (4).

(Woosley et al. 1973)

$\rho(t)=\rho_{\text {peak }} e^{-t / \tau_{H D}}$,

$T_{9}(t)=T_{9, \text { peak }}\left(\frac{\rho(t)}{\rho_{\text {peak }}}\right)^{\gamma-1}$.

Here $\gamma$ denotes the adiabatic exponent and $\tau_{\mathrm{HD}}=446 \chi \rho_{\text {peak }}^{-1 / 2}$ is the hydrodynamical timescale. With the help of the scaling parameter $\chi$, the dependence of the results on the timescale can be examined.

Meakin et al. (2009) present an updated prescription for the density evolution in SNe Ia. They employ an exponential temperature decay and ensure adiabaticity by fixing the entropy to the post-burning state. The entropy $S$ is a function of temperature, density, and averaged values of the mass number $\bar{A}$ and the proton number $\bar{Z}$. Thus, the density can be obtained from $S=S(T(t), \rho(t), \bar{A}, \bar{Z})$. However, for demonstration purposes we stick to the formulation of Woosley et al. (1973) in the following since the main statements about freeze-out from NSE are not affected by the exact choice of the expansion prescription.

Incomplete silicon burning is characterized by the presence of two quasi-equilibrium clusters, i.e., only certain regions in the chart of nuclei that have reached equilibrium states, centered around ${ }^{28} \mathrm{Si}$ and ${ }^{56} \mathrm{Ni}$, respectively. They are separated by the bottleneck at a mass number of $A \approx 45$ (e.g., Ti and Sc). These elements are weakly bound compared to $\mathrm{Ca}$ and are therefore low in abundance. Hence, there is only little flow of material through the bottleneck and intermediate-mass elements (IMEs), and IGEs remain present after the burning is quenched. At higher temperatures the bottleneck is removed. The equilibrium clusters merge and matter achieves the state of NSE, ultimately converting silicon to IGEs almost completely. The temperature necessary for silicon exhaustion, $X\left({ }^{28} \mathrm{Si}\right) \lesssim 5 \times 10^{-3}$, can be approximated by (Woosley et al. 1973)

$T_{9, \text { peak }} \gtrsim 4.3\left(\frac{\rho_{\text {peak }}}{\chi^{2}}\right)^{1 / 68}$.

In NSE, the abundance distribution is uniquely determined by density, temperature, and electron fraction $Y_{e}$. The composition changes as soon as the first reactions drop out of equilibrium due to decreasing temperature. At high densities matter is characterized by a low fraction of light and of free particles such as neutrons, protons, and $\alpha$-particles. Therefore, the composition during this normal freeze-out or particle-poor freeze-out is not altered significantly by the capture of light particles during expansion. At lower densities, in contrast, light particles are more abundant and thus react with the prevailing iron group nuclei and bring matter out of NSE composition. Due to the high mass fraction of $\alpha$-particles, this drop-out of equilibrium is called alpha-rich freeze-out. Woosley et al. (1973) derive the approximate relation

$\rho_{\text {peak }} \lesssim \min \left\{\begin{array}{l}4.5 \times 10^{5} T_{9, \text { peak }}^{3} \\ 2.5 \times 10^{5} T_{9, \text { peak }}^{4} \chi^{-2 / 3}\end{array}\right.$

for the density separating $\alpha$-rich freeze-out and normal freezeout using $\gamma=4 / 3$ in Eqs. (1) and (2).

Therefore, the $T_{\text {peak }}-\rho_{\text {peak }}$-plane is split into three regions by Eqs. (3) and (4), as illustrated in Fig. 1 (see also Fig. 20 of Woosley et al. 1973). These regions produce different chemical compositions after the last reactions have frozen out. This is also visualized in Fig. 2 for the different explosion models explained in the next section. The separation of the different regions can be shifted continuously by a variation of the scaling parameter $\chi$. The gray shaded areas in Figs. 1 and 2 cover values of $\chi$ between 1 and 10.

\subsection{Neutronization in high-density material}

We discuss in the previous section how the NSE composition is altered in the two different freeze-out regimes, but the NSE composition itself is determined by the electron fraction $Y_{e}$ (related to the neutron excess $\eta=1-2 Y_{e}$ ). In NSE, but also in quasistatistical equilibrium (QSE), the most abundant nuclei after freeze-out are those with the highest binding energy and an electron fraction close to that of the initial fuel. For symmetric matter (i.e., $Y_{e}=0.5$ ) the most abundant nucleus is ${ }^{56} \mathrm{Ni}$. As $Y_{e}$ decreases, isotopes like ${ }^{57-60} \mathrm{Ni}$ and ${ }^{54-58} \mathrm{Fe}$ become more abundant depending on the actual electron fraction and their binding energies. The value of $Y_{e}$ is determined and altered by three mechanisms.

First, the electron fraction of the WD is set by the metallicity $Z$ of the progenitor main sequence star. Most important are the abundances of ${ }^{56} \mathrm{Fe}$ and $\mathrm{CNO}$ nuclei which are mostly converted to ${ }^{14} \mathrm{~N}$ in hydrogen burning (CNO cycle) and subsequently to ${ }^{22} \mathrm{Ne}$ in helium burning. These two isotopes, ${ }^{56} \mathrm{Fe}$ and ${ }^{22} \mathrm{Ne}$, provide the dominant part of the surplus of neutrons in the exploding WD. Timmes et al. (2003) derive an approximate relation for the mass of ${ }^{56} \mathrm{Ni}$ as a function of metallicity. It shows a decreasing trend for ${ }^{56} \mathrm{Ni}$ with increasing $\mathrm{Z}$, and a variation of $25 \%$ if $\mathrm{Z}$ is varied by a factor of three.

Second, the initial $Y_{e}$ of the WD is altered during convective carbon burning ("simmering") preceding the thermonuclear runaway in a $M_{\mathrm{Ch}}$ WD. The number of neutrons is increased via the capture of free electrons onto the highly ionized atoms (Bahcall 1964):

$e^{-}+(Z, A) \rightarrow(Z-1, A)+v$.

These endoergic reactions require high electron energies, and therefore only become important at high densities. Chamulak et al. (2008) study the behavior of $Y_{e}$ during carbon burning and find that the reaction chain $\left.{ }^{12} \mathrm{C}(p, \gamma)\right)^{13} \mathrm{~N}\left(e^{-}, v\right)^{13} \mathrm{C}$ is 


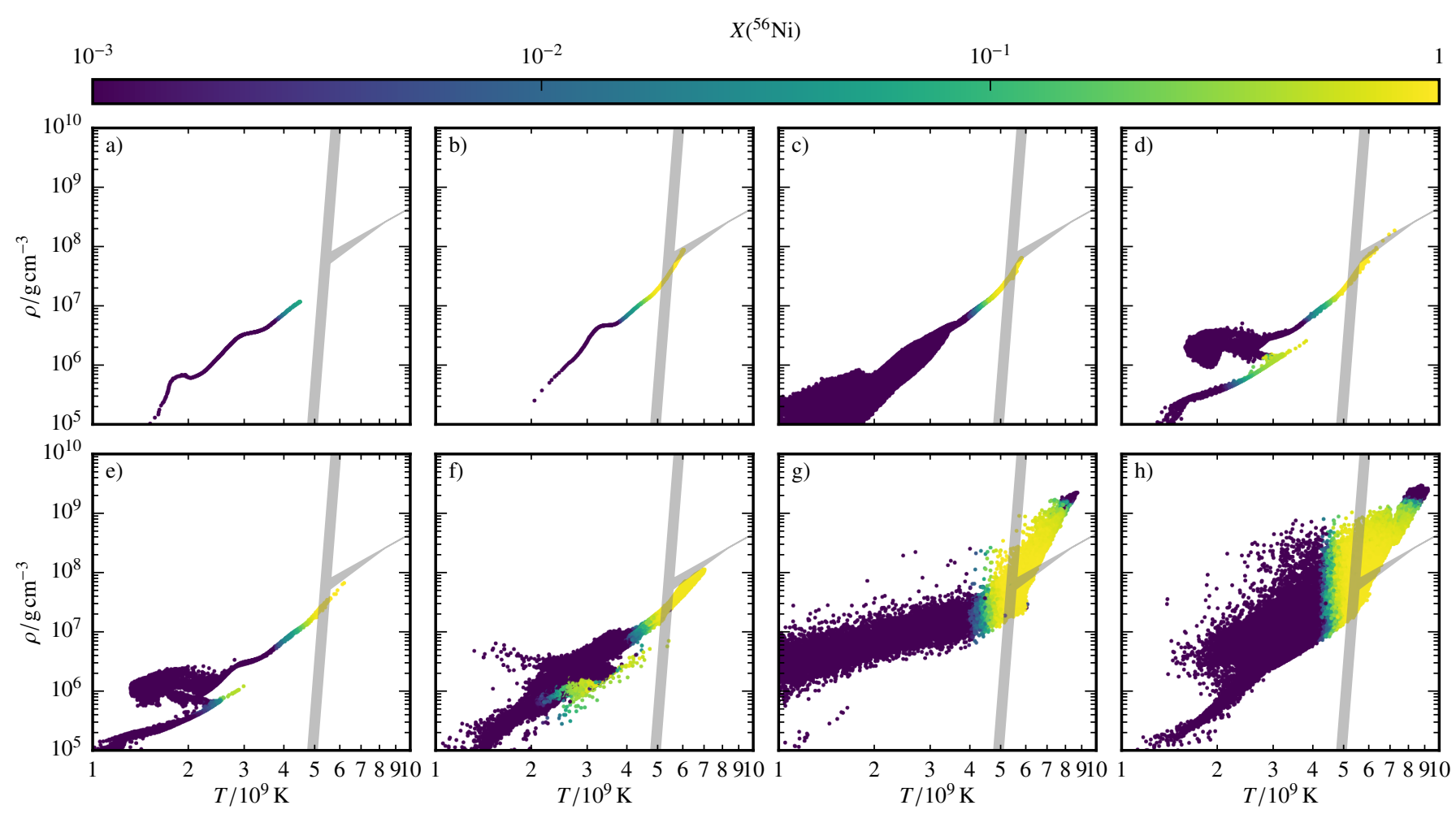

Fig. 2. Distribution of the tracer particles in the $T_{\text {peak }}-\rho_{\text {peak }}$ plane with color-coded mass fraction of ${ }^{56} \mathrm{Ni} 100 \mathrm{~s}$ after triggering the explosion. The panels show (a) PD081, $(b)$ PD115, $(c)$ VM, $(d)$ CSDD-S, $(e)$ CSDD-L, $(f)$ M2a $\mathrm{a}_{\odot},(g)$ R60, $(h)$ N100ddt. The gray shaded regions separate incomplete (left) from complete silicon burning (right) and normal freeze-out (upper right) from $\alpha$-rich freeze-out (lower right) according to Eqs. (3) and (4). The area covered corresponds to a varying scaling parameter: $1<\chi<10$.

the dominant mechanism for reducing $Y_{e}$ for densities of approximately $1 \times 10^{9} \mathrm{~g} \mathrm{~cm}^{-3}$. The electron capture on ${ }^{13} \mathrm{~N}$ is gradually replaced by ${ }^{12} \mathrm{C}\left({ }^{12} \mathrm{C}, p\right)^{23} \mathrm{Na}\left(e^{-}, v\right)^{23} \mathrm{Ne}$ for $\rho>1.7 \times 10^{9} \mathrm{~g} \mathrm{~cm}^{-3}$. Chamulak et al. (2008) estimate a maximum reduction in $Y_{e}$ by $6.3 \times 10^{-4}$, and Piro \& Bildsten (2008) suggest a carbondependent value of $\left|\Delta Y_{e, \max }\right|=1.7 \times 10^{-3} X\left({ }^{12} \mathrm{C}\right)$.

Finally, the most dramatic changes to the electron fraction happen during the explosion itself. In NSE, electrons are mostly captured by free protons at high temperatures. Subsequently, as the temperature drops, IGEs capture most of the electrons. This leads to a decrease in $Y_{e}$ to $Y_{e} \approx 0.44$ in the most extreme cases in the central regions of the exploding WD star. Therefore, the regions with the highest density do not contribute significantly to the production of ${ }^{56} \mathrm{Ni}$ (Brachwitz et al. 2000), but to more neutron-rich IGEs. In general, the nucleosynthesis results then depend on the central density and the corresponding density gradient. Detailed studies of the nucleosynthesis in Chandrasekhar-mass models and the effect of neutronization have been carried out by Thielemann et al. (1986), Iwamoto et al. (1999), and, with updated electron capture rates (Langanke \& Martinez-Pinedo 2000), by Brachwitz et al. (2000) and Bravo (2019). When 3D effects in deflagration models are taken into account the final abundance stratification are smoothed out compared to the theoretical prediction based on density, metallicity, and neutronization (Seitenzahl et al. 2013a).

In summary, the neutronization due to electron capture is an effect restricted to the high densities reached only in WDs close to $M_{\mathrm{Ch}}$, and hence the abundances of very neutron-rich isotopes are a hint to the Chandrasekhar-mass scenario. The abundance ratio of nickel to iron, for instance, measured in late-time spectra of SNe Ia can be taken as a proxy for the quotient ${ }^{58} \mathrm{Ni} /{ }^{56} \mathrm{Ni}$, and thus for neutronization. This is used by Flörs et al. (2020) to infer the contribution of sub- $M_{\mathrm{Ch}}$ progenitors to $\mathrm{SNe}$ Ia in relation to $M_{\mathrm{Ch}}$ progenitors.

\subsection{Explosive helium burning}

In addition to explosive silicon burning, the burning of helium is another source of nucleosynthesis products in the doubledetonation scenario (see Sect. 1). Explosive helium burning was studied by Khokhlov (1984) and Khokhlov \& Érgma (1985). They found that in general the burning is characterized by a competition between the triple- $\alpha$ reaction and $\alpha$-captures on heavier nuclei. First, ${ }^{12} \mathrm{C}$ is synthesized by the reaction $3 \alpha \rightarrow{ }^{12} \mathrm{C}$, and subsequently heavier $\alpha$-elements $\left({ }^{16} \mathrm{O},{ }^{20} \mathrm{Ne},{ }^{24} \mathrm{Mg},{ }^{28} \mathrm{Si},{ }^{32} \mathrm{~S}\right.$, $\left.{ }^{36} \mathrm{Ar},{ }^{40} \mathrm{Ca},{ }^{44} \mathrm{Ti}, . ..\right)$ are produced. The timescale for the capture of an $\alpha$-particle increases for higher mass numbers $A$ due to the higher Coulomb barriers. These are penetrated more easily at higher particle energies, and the timescale is therefore temperature dependent. Consequently, the $\alpha$-chain stops as soon as the timescale for the triple- $\alpha$-reaction is shorter than for the next $\alpha$-capture. However, above a certain density-dependent temperature of approximately $2 \times 10^{9} \mathrm{~K}$ at $\rho=5 \times 10^{6} \mathrm{~g} \mathrm{~cm}^{-3},{ }^{56} \mathrm{Ni}$ is always the most abundant isotope since it has the highest binding energy at $Y_{e}=0.5$ (see Sect. 2.1). These temperatures are usually surpassed in helium detonations, and therefore most of the material is converted to ${ }^{56} \mathrm{Ni}$. Nevertheless, there is a way to stop the $\alpha$-chain before ${ }^{56} \mathrm{Ni}$ is reached, even at high temperatures. If the initial fuel is polluted with carbon, oxygen, or nitrogen, for instance, the slow triple- $\alpha$ reaction is bypassed by $\alpha$-capture on these seed nuclei. This leads to a very fast depletion of $\alpha$-particles and the nucleosynthesis stops below $A=56$ once the material runs out of $\alpha$-particles (see also Woosley $\&$ Kasen 2011, Shen \& Moore 2014; Gronow et al. 2020). 
Beyond $A=56$, the reverse reactions become increasingly important and start to balance the $\alpha$-captures to some extent. Nevertheless, a high abundance of $\alpha$-particles results in an enhanced production of elements beyond $\mathrm{Ni}$, such as $\mathrm{Cu}$ and $\mathrm{Zn}$, compared to their NSE abundance. The timescale to reach NSE is about $1 \mathrm{~s}$ at a temperature of $5 \times 10^{9} \mathrm{~K}$. However, conditions necessary for NSE are not achieved in most of the helium detonations, and thus nucleosynthesis exhibits interesting differences in the burning products of the $\mathrm{CO}$ core.

\subsection{Models}

In this work we investigate three distinct kinds of explosion models. The first class consists of pure detonations of subChandrasekhar mass CO WDs. We closely examine the violent merger $(\mathrm{VM})$ of a $0.9 M_{\odot}$ with a $1.1 M_{\odot}$ WD simulated by

Pakmor et al. (2012a). In addition, two pure detonations of CO WDs with total masses of the progenitor of 0.81 (PD081) and $1.15 M_{\odot}$ (PD115) of Sim et al. (2010) are included in our analysis.

We then study models including a detonation of a helium shell on top of a sub- $M_{\mathrm{Ch}}$ mass WD (also labeled ".Ia-SN", Bildsten et al. 2007; Shen \& Bildsten 2009; Shen et al. 2010) eventually triggering a second detonation burning the $\mathrm{CO}$ core (double detonation). Two models are taken from Sim et al. (2012) that follow the explosion of low-mass CO cores $(0.58$ and $0.45 M_{\odot}$ ) with a prominent helium shell of $0.21 M_{\odot}$ (hereafter CSDD-S and CSDD-L, respectively). The second detonation is triggered via the converging shock mechanism not far from the center of the WD (Fink et al. 2007). The HeD-S model follows the same setup as CSDD-S, but the detonation of the core is suppressed. These pure helium detonations are a possible explosion mechanism for Ca-rich transients (e.g., Inserra et al. 2015). Moreover, a double detonation in the core of a $1.05 M_{\odot}$ WD with a carbon-enriched helium shell of $0.073 M_{\odot}$ (Model M2a of Gronow et al. 2020) is included. In this case, a detonation in the core is triggered at its outer edge when the helium detonation front converges on the far side of its ignition. In addition, an equivalent model to M2a at solar metallicity $M 2 \mathrm{a}_{\odot}$ is investigated. This model is set up as a WD with a total mass of $1.06 M_{\odot}$. Its core consists of ${ }^{12} \mathrm{C},{ }^{16} \mathrm{O}$, and, to reproduce the metallicity, $1.34 \%$ of ${ }^{22} \mathrm{Ne}$. In the ${ }^{4} \mathrm{He}$ shell $\left(M_{\text {shell }}=0.075 M_{\odot}\right)$ an admixture of $0.34 \%$ by mass of ${ }^{14} \mathrm{~N}$ accounts for solar metallicity in the hydrodynamical explosion simulation.

Finally, the $M_{\mathrm{Ch}}$ scenario is analyzed. We add the N5def model of Fink et al. (2014) as an example of a pure deflagration, the N100ddt delayed detonation identical with model N100 of Seitenzahl et al. (2013a), as well as a pure deflagration in a $M_{\mathrm{Ch}}$ WD with central density of $2.6 \times 10^{9} \mathrm{~g} \mathrm{~cm}^{-3}$. The latter $(\mathrm{R} 60)$ is ignited in a single spot $60 \mathrm{~km}$ off-center and, like N5def, does not disrupt the whole star but leaves behind a bound remnant. The model produces a very faint explosion ejecting only $0.018 M_{\odot}$ of ${ }^{56} \mathrm{Ni}$ and $0.049 M_{\odot}$ of material in total. Moreover, the kinetic energy of the ejecta amounts to $8.17 \times 10^{48} \mathrm{erg}$ and an intact WD of $1.33 M_{\odot}$ is left behind after the explosion. This model has been calculated for this paper with methods similar to Fink et al. (2014) but with an updated equation of state (Timmes \& Arnett 1999) and a gravity solver based on fast Fourier transforms.

More details concerning the individual setups and the employed codes can be found in the references above. Moreover, data for the models VM, CSDD-S, CSDD-L, HeD-S, N5def, N100ddt, and M2a have been made publicly available in the online model database HESMA (Kromer et al. 2017). It should be noted that all hydrodynamic explosion simulations were done using the LEAFS code (Reinecke et al. 1999, 2002; Röpke 2005) except for the double detonations M2a and M2a $\mathrm{a}_{\odot}$, which were computed with AREPO (Springel 2010).

Because solving a large nuclear network in parallel with the actual explosion simulation goes beyond the scope of the current computational resources, the nucleosynthesis yields are calculated in a post-processing step. To this end, virtual tracer particles are placed into the exploding WD star and advected passively with the fluid flow recording their thermodynamic history. Subsequently, this data is used to determine the isotopic abundances produced by the explosion.

Because we partially work with models from HESMA but also add new explosion simulations, the postprocessing and the treatment of metallicity in it follows different approaches: Models CSDD-L, CSDD-S, HeD-S, PD081, and PD115 were postprocessed at zero metallicity and VM, N100ddt, and N5def at solar metallicity using the methods described in Travaglio et al. (2004). In the latter models, solar metallicity was mimicked by including $2.5 \%$ of ${ }^{22} \mathrm{Ne}$ to approximately adjust $Y_{e}$ to the solar value (see also Seitenzahl et al. 2013a). All other simulations, i.e., M2a, M2 $\mathrm{a}_{\odot}$, and R60, use the YANN code (Pakmor et al. $2012 b$ ) for postprocessing. The metallicity of the material in M2a is assumed to be zero, while M2 $\mathrm{a}_{\odot}$ and R60 apply solar metallicity. To this end, the abundances of all isotopes in the core material (carbon and oxygen) are set according to Asplund et al. (2009) except for elements lighter than fluorine. While $\mathrm{H}$ and $\mathrm{He}$ are ignored, all $\mathrm{CNO}$ isotopes are converted to ${ }^{22} \mathrm{Ne}$, thus accounting for their processing in $\mathrm{H}$ and $\mathrm{He}$ burning. In the shell material of $\mathrm{M} 2 \mathrm{a}_{\odot}$ carbon and oxygen are instead converted to ${ }^{14} \mathrm{~N}$. To check the effect of the different metallicity implementations we also post-processed $\mathrm{R} 60$ and $\mathrm{M} 2 \mathrm{a}_{\odot}$ with the appropriate amounts of ${ }^{22} \mathrm{Ne}$ in $\mathrm{CO}$ material and ${ }^{14} \mathrm{~N}$ in the helium shell, but no other isotopes present. These variants are labeled $\mathrm{R} 60_{\mathrm{Ne}}$ and M2 $\mathrm{a}_{\mathrm{Ne}}$. In all cases the 384 species network of Travaglio et al. (2004) is utilized. Reaction rates were taken from the REACLIB database (Rauscher \& Thielemann 2000, updated 2009) and only for our most recent simulations (e.g., R60, M2a) the version of 2014 is applied.

\section{Discussion of the nucleosynthesis yields}

An instructive way to illustrate the burning regimes reached by a specific model is the distribution of the tracer particles in the $T_{\text {peak }}-\rho_{\text {peak }}$ diagram shown in Fig. 1 .

In the case of the sub-Chandrasekhar mass pure detonations (VM, PD081, PD115) the important characteristic from the nucleosynthesis point of view is the low central density $\left(\rho_{c} \lesssim 10^{8} \mathrm{~g} \mathrm{~cm}^{-3}\right)$ of the WD. Therefore, IGEs are produced in alpha-rich freeze-out and incomplete silicon burning only (see Fig. 2). While the higher-mass CO core (PD115) produces ${ }^{56} \mathrm{Ni}$ also in NSE ( $\alpha$-rich freeze-out), the low-mass core model (PD081) synthesizes all of its ${ }^{56} \mathrm{Ni}$ in incomplete silicon burning. In addition, the fuel in the VM model stays below densities of $8 \times 10^{7} \mathrm{~g} \mathrm{~cm}^{-3}$, and therefore it burns to NSE in the $\alpha$-rich freeze-out regime. In the merger process the structure of the primary is not much affected, but the disruption of the secondary causes the larger scatter in the thermodynamic properties of the tracer particles at lower densities (see Fig. 2).

In addition to this, the double-detonation models (CSDD-S, CSDD-L, M2 $\mathrm{a}_{\odot}$ ) clearly show that ${ }^{56} \mathrm{Ni}$ is also produced in the helium shell detonation. The tracer particles located in the shell cover an area in the parameter space shown in Fig. 2 that is slightly below that of the core detonation. The scatter in the $M_{\mathrm{Ch}}$ deflagration models is larger due to the turbulent 


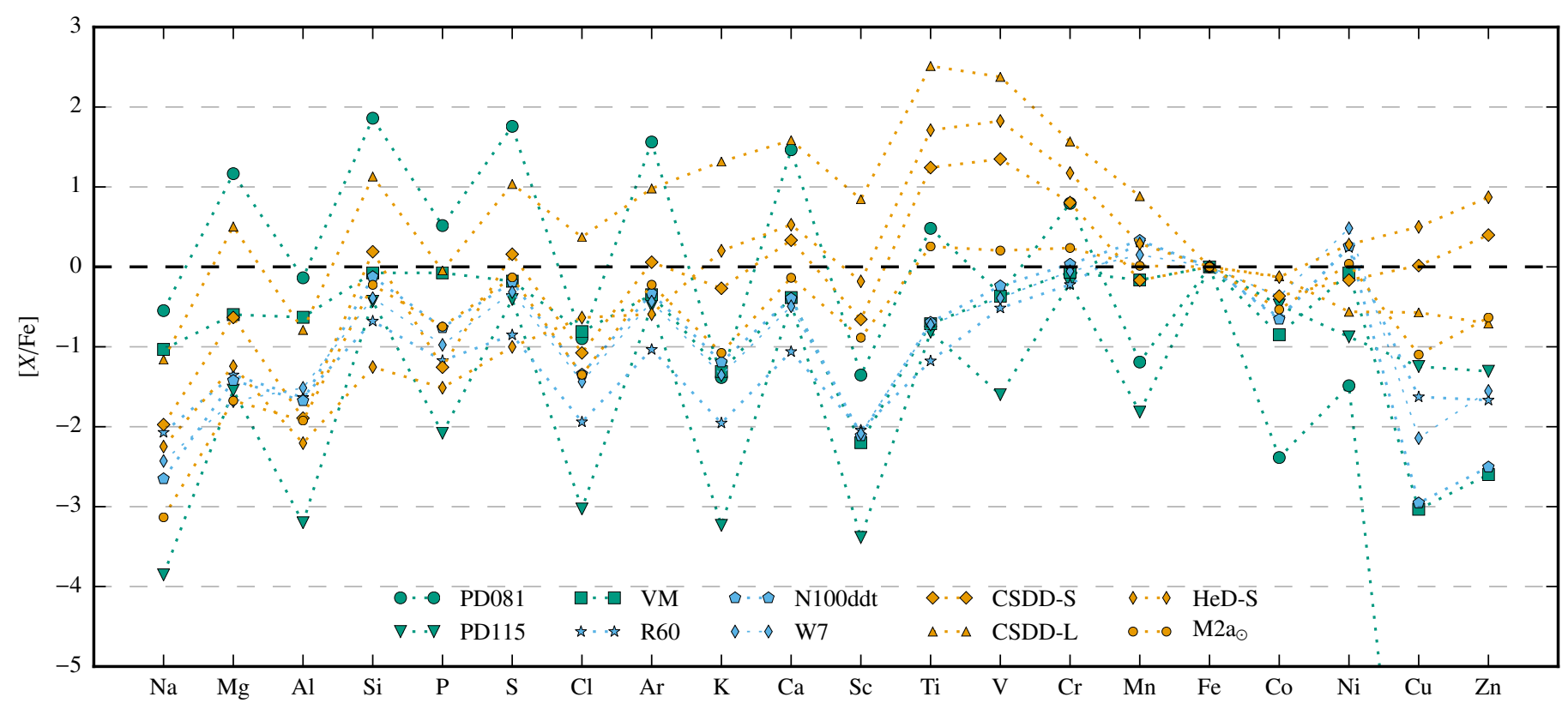

Fig. 3. Elemental ratios to $\mathrm{Fe}$ (with radioacitve isotopes decayed to $2 \times 10^{9} \mathrm{yr}$ ) compared to their solar ratios for three sub- $M_{\mathrm{Ch}}$ detonations $(\mathrm{VM}$, PD081, PD115), three double detonations (M2 $\mathrm{a}_{\odot}$, CSDD-S, CSDD-L), one helium detonation (HeD-S), two pure $M_{\mathrm{Ch}}$ deflagrations (R60, W7), and a delayed detonation (N100ddt).

motion of the flame and the pre-expansion of the WD during the burning phase. This is even more obvious in the delayed detonation model since some tracers might be affected first by the deflagration and subsequently by the detonation. We note that for the single-spot ignited Model R60 the WD is not disrupted completely, and hence Fig. 2 only shows ejected tracers. Because only such massive WDs reach high densities of $\rho \gtrsim 10^{9} \mathrm{~g} \mathrm{~cm}^{-3}$ and consequently their nucleosynthesis yields originate mainly from normal freeze-out, these kinds of explosions contribute to elements not synthesized in sub- $M_{\mathrm{Ch}}$ models.

The gross nucleosynthesis yields are summarized in Fig. 3, which displays the elemental ratio to iron compared to the solar ratio according to Asplund et al. (2009). Since the PD081 model does not burn to NSE, it synthesizes super-solar amounts of IMEs showing a strong odd-even effect. Furthermore, it exhibits a rather high $\mathrm{Cr}$ abundance and drops off steeply for higher mass IGEs. CSDD-S also produces super-solar abundances of some IMEs, such as $\mathrm{Si}, \mathrm{S}$, $\mathrm{Ar}$, and $\mathrm{Ca}$ which is not surprising as it shares the characteristics of a low-mass CO core with the PD081 model. However, the helium shell detonation adds substantial amounts of light IGEs ( $\mathrm{Ti}, \mathrm{V}, \mathrm{Cr}$ ) as well as $\mathrm{Cu}$ and $\mathrm{Zn}$ to the mix. These elements are most abundant in HeD-S since they are primarily produced in the helium detonation. Moreover, it should be noted that HeD-S and CSDD-L yield super-solar amounts of Mn. The VM yields are rather inconspicuous for not showing any overproduction, but instead significant underproduction of $\mathrm{Mn}, \mathrm{Co}, \mathrm{Cu}$, and $\mathrm{Zn}$. Interestingly, the $\mathrm{M} 2 \mathrm{a}_{\odot}$ simulation exhibits characteristics similar to those seen in pure detonations (a strong odd-even effect for IMEs, an underproduction of Co, a drop-off for $\mathrm{Cu}$ and $\mathrm{Zn}$ ) as well as helium shell detonation features (supersolar values of $\mathrm{Ti}, \mathrm{V}, \mathrm{Cr}$ and solar abundance of $\mathrm{Mn}$ ). Finally, the pure deflagration explosions R60 and W7 (we included the W7 model from Iwamoto et al. 1999 because it has been widely used in GCE calculations) and the delayed detonation N100ddt display low abundances of the light IGEs $\mathrm{V}$ and $\mathrm{Cr}$ and supersolar abundances of $\mathrm{Mn}$ and stable Ni. Moreover, the produced amounts of $\mathrm{Cu}$ and $\mathrm{Zn}$ are negligible, as is also the case for the pure detonations VM and PD081.

We summarize that all SNe Ia models included here underproduce Co compared to the solar value. Moreover, we confirm the known facts that $M_{\mathrm{Ch}}$ explosions can produce $\mathrm{Mn}$ at supersolar values and that they also overproduce stable $\mathrm{Ni}$. These have been used to discriminate between $M_{\mathrm{Ch}}$ and sub- $M_{\mathrm{Ch}}$ explosions in nebular spectra (Flörs et al. 2020). The theoretical reason for this distinction is that stable $\mathrm{Ni}\left({ }^{58,60} \mathrm{Ni}\right)$ is produced at higher densities due to the lower electron fraction (see Sect. 2.2). However, we also find the double-detonation models can overproduce $\mathrm{Mn}$ and $\mathrm{Ni}$, which makes the nucleosynthetic distinction between $M_{\mathrm{Ch}}$ and sub- $M_{\mathrm{Ch}}$ explosions not as straightforward as expected. The lighter elements $\mathrm{Ti}, \mathrm{V}$, and $\mathrm{Cr}$ stem either from $\mathrm{He}$ detonations or from the incomplete silicon burning region in low-mass $\mathrm{CO}$ cores. The heaviest IGEs $\mathrm{Cu}$ and $\mathrm{Zn}$ are synthesized in He detonations only, and therefore test the double-detonation scenario: any observations and GCE calculations finding $\mathrm{Cu}$ or $\mathrm{Zn}$ to be produced by $\mathrm{SNe}$ Ia would hint to the occurrence of this explosion mechanism.

\subsection{Manganese}

The only stable isotope of $\mathrm{Mn},{ }^{55} \mathrm{Mn}$, is produced in CCSNe and in $\mathrm{SNe}$ Ia in incomplete silicon burning primarily via the channel ${ }^{55} \mathrm{Co} \rightarrow{ }^{55} \mathrm{Fe} \rightarrow{ }^{55} \mathrm{Mn}$. As already pointed out by Seitenzahl et al. (2013b), a super-solar production of $\mathrm{Mn}$ is required to explain the rise in $[\mathrm{Mn} / \mathrm{Fe}]$ for $[\mathrm{Fe} / \mathrm{H}] \gtrsim-1$ to its solar value. Although the CCSN contribution to $\mathrm{Mn}$ is uncertain, all current models predict $[\mathrm{Mn} / \mathrm{Fe}]$ ratios below the solar value. Therefore, $M_{\mathrm{Ch}}$ explosions must be added to the mix of SNe Ia. Only these WDs reach densities high enough for normal freeze-out, and thus offer an additional site of production to the regime of incomplete silicon burning. The production of ${ }^{55} \mathrm{Co}$ is illustrated in Fig. 4 for the pure deflagration model R60, the violent merger model VM, and the double-detonation model $\mathrm{M} 2 \mathrm{a}_{\odot}$. It clearly shows that ${ }^{55} \mathrm{Co}$ is synthesized in normal 


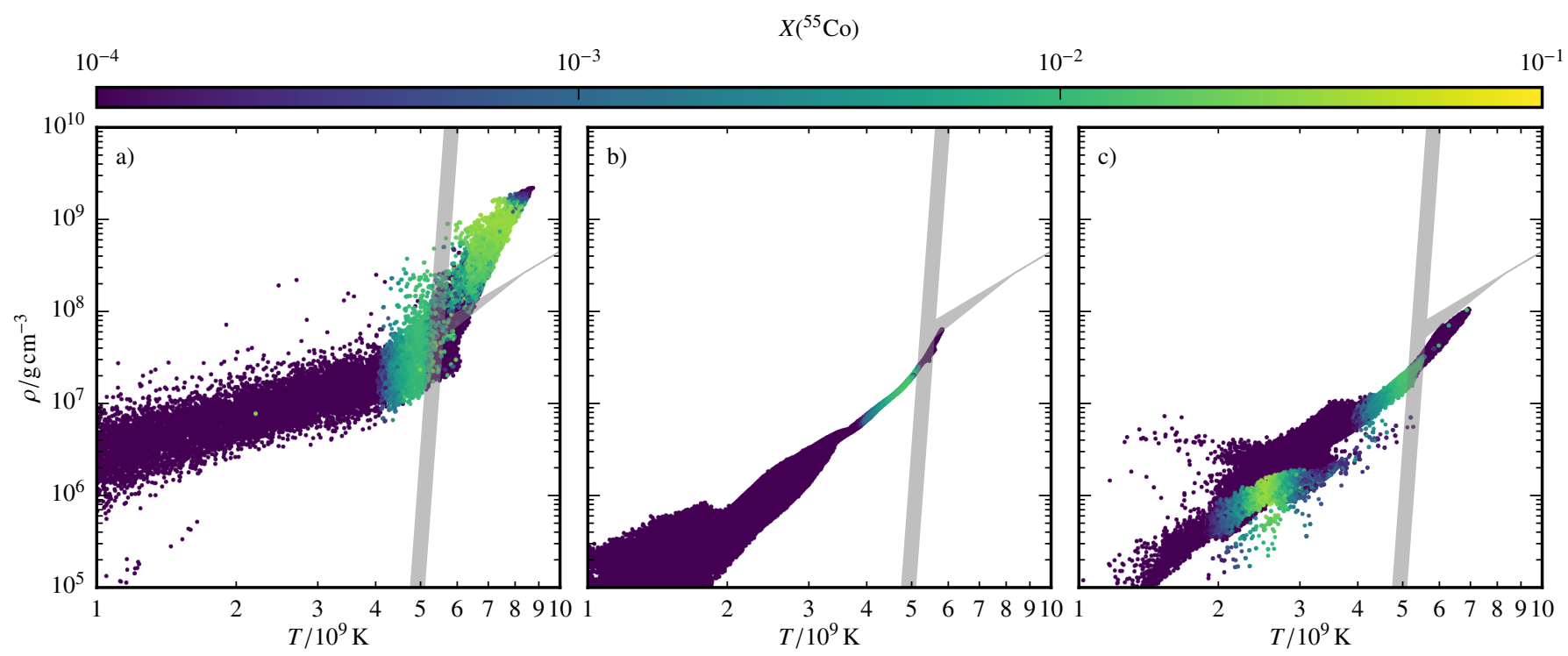

Fig. 4. Same as Fig. 2 with a color-coded mass fraction of ${ }^{55}$ Co. The panels show (a) R60, (b) VM, (c) M2a $\mathrm{a}_{\odot}$.

Table 1. Mn-to-Fe ratio compared to solar for the total explosion at $100 \mathrm{~s}$ after ignition and to shell and core material only, the total amount of stable $\mathrm{Mn}$ in $M_{\odot}$, and the radioactive and stable isotopes from which ${ }^{55} \mathrm{Mn}$ originates (shown as percentages).

\begin{tabular}{lccccccc}
\hline \hline Model & {$[\mathrm{Mn} / \mathrm{Fe}]$} & {$[\mathrm{Mn} / \mathrm{Fe}]_{\text {shell }}$} & {$[\mathrm{Mn} / \mathrm{Fe}]_{\text {core }}$} & $\mathrm{Mn}$ & ${ }^{55} \mathrm{Co}$ & ${ }^{55} \mathrm{Fe}$ & ${ }^{55} \mathrm{Mn}$ \\
\hline PD081 & -1.19 & - & - & $4.90 \mathrm{e}-06$ & 99.3 & 0.7 & - \\
PD115 & -1.82 & - & - & $1.04 \mathrm{e}-06$ & 97.6 & 2.4 & - \\
VM & -0.16 & - & - & $3.74 \mathrm{e}-03$ & 97.9 & 2.1 & - \\
M2a $\odot$ & -0.03 & 0.81 & -0.07 & $4.57 \mathrm{e}-03$ & 98.9 & 1.1 & - \\
M2a & -0.15 & 0.81 & -0.21 & $3.46 \mathrm{e}-03$ & 99.5 & 0.5 & - \\
M2a & -1.00 & 0.53 & -1.63 & $4.94 \mathrm{e}-04$ & 99.7 & 0.3 & - \\
CSDD-L & 0.88 & 1.69 & -1.19 & $1.47 \mathrm{e}-03$ & 99.6 & 0.4 & - \\
CSDD-S & -0.17 & 0.29 & -1.37 & $1.27 \mathrm{e}-03$ & 99.9 & 0.1 & - \\
HeD-S & 0.29 & 0.29 & - & $1.21 \mathrm{e}-03$ & 99.9 & 0.1 & - \\
N5def & 0.35 & - & - & $4.03 \mathrm{e}-03$ & 87.5 & 12.3 & 0.2 \\
N100ddt & 0.33 & - & - & $1.33 \mathrm{e}-02$ & 85.8 & 14.0 & 0.2 \\
R60 & 0.11 & - & - & $2.30 \mathrm{e}-04$ & 85.0 & 14.8 & 0.2 \\
R60 & 0.11 & - & - & $2.30 \mathrm{e}-04$ & 85.1 & 14.7 & 0.2 \\
W7 & 0.16 & - & - & $8.87 \mathrm{e}-03$ & - & - & - \\
\hline
\end{tabular}

freeze-out in the R60 model in contrast to VM and M2a $\mathrm{a}_{\odot}$. Furthermore, it can be seen that Mn also originates from the He detonation in Model M2 $\mathrm{a}_{\odot}$. Table 1 summarizes the $\mathrm{Mn}$ yields and also gives the fraction of the isotopes contributing to the final $\mathrm{Mn}$ abundance. It reveals that only $M_{\mathrm{Ch}}$ deflagrations (N5def, N100ddt, R60, R60 ${ }_{\mathrm{Ne}}$, W7), the double detonation CSDD-L, and the helium detonation HeD-S achieve super-solar $[\mathrm{Mn} / \mathrm{Fe}]$ ratios. For model R60 the $[\mathrm{Mn} / \mathrm{Fe}]$ values are somewhat lower compared to N100ddt and N5def due to the newer set of reaction rates employed in the postprocessing. While $\mathrm{M} 2 \mathrm{a}_{\odot}$ roughly reaches the solar value of [Mn/Fe], the other double detonations (M2a, M2 $\mathrm{a}_{\mathrm{Ne}}$ and CSDD-S) exhibit a sub-solar production of [ $\mathrm{Mn} / \mathrm{Fe}]$.

The value of $[\mathrm{Mn} / \mathrm{Fe}]$ in the double-detonation models is governed by three fundamental parameters of the initial model:

(i) The ratio of shell to core mass $\left(M_{\text {shell }} / M_{\text {core }}\right)$ is a crucial factor. Most $\mathrm{Mn}$ is produced in the helium detonation which is reflected by the super-solar value of 0.29 for HeD-S (see Table 1). In contrast to that $[\mathrm{Mn} / \mathrm{Fe}]$ is below solar in CSDD-S due to the sub-solar value of the core detonation. The WD in Model CSDD-L, however, has a very low core mass $\left(0.45 M_{\odot}\right)$ and a helium shell of $0.2 M_{\odot}$ (the same as CSDD-S), and therefore the contribution of the core detonation to $[\mathrm{Mn} / \mathrm{Fe}]$ is less significant.

(ii) The density of the helium shell also affects $[\mathrm{Mn} / \mathrm{Fe}]$. ${ }^{55} \mathrm{Co}$ is produced in a well-defined range of initial densities in the helium envelope above approximately $6 \times 10^{5} \mathrm{~g} \mathrm{~cm}^{-3}$. The density at the base of the helium shell for the low-mass progenitors in CSDD-L $\left(\rho_{\mathrm{c}}=5.92 \times 10^{5} \mathrm{~g} \mathrm{~cm}^{-3}\right)$ and for CSDD-S $\left(\rho_{\mathrm{c}}=12 \times 10^{5} \mathrm{~g} \mathrm{~cm}^{-3}\right)$ is sufficient to synthesize ${ }^{55} \mathrm{Co}$. Thus, the amount of Mn is quite similar in both models. However, the amount of $\mathrm{Fe}$ is much lower in CSDD-L due to the lower density in the envelope, and therefore the discrepancy between CSDD-L and CSDD-S in $[\mathrm{Mn} / \mathrm{Fe}]$ mainly originates from the yields of the shell detonation. Model CSDD-L gives a value of 1.69 for $[\mathrm{Mn} / \mathrm{Fe}]$ from the helium shell detonation compared to 0.29 in Model CSDD-S.

(iii) The progenitor metallicity plays an essential role for the production of $\mathrm{Mn}$.

Following argument (i) from above, a very low value of $[\mathrm{Mn} / \mathrm{Fe}]$ could be expected for Model $\mathrm{M} 2 \mathrm{a}_{\odot}$ because of the 
low-mass helium shell and the $\sim 1 M_{\odot}$ core. The data, in contrast, show an approximately solar value. The reason is that it has been calculated at solar metallicity, which leads to $[\mathrm{Mn} / \mathrm{Fe}]_{\text {core }}=-0.07$ (comparable to Model VM) for core material and $[\mathrm{Mn} / \mathrm{Fe}]_{\text {shell }}=0.81$ for the shell. Since the production of nuclei more neutron-rich than ${ }^{56} \mathrm{Ni}$ is enhanced for lower $Y_{e}$, this shift in $[\mathrm{Mn} / \mathrm{Fe}]$ is reasonable. The yields of model $\mathrm{M} 2 \mathrm{a}_{\mathrm{Ne}}$ fall into line with this analysis. The $[\mathrm{Mn} / \mathrm{Fe}]$ value in the core material slightly decreases, however, indicating a low dependence on the initial distribution of nuclei. Furthermore, Model M2a at zero metallicity from Gronow et al. (2020) gives $[\mathrm{Mn} / \mathrm{Fe}]=-1.00$, which supports the explanations given above.

It is generally found that $\mathrm{CO}$ detonations at zero metallicity (also the core detonations of CSDD-L and CSDD-S) produce less Mn than models at higher metallicity (VM, M2 $\left.\mathrm{a}_{\odot}, \mathrm{M} 2 \mathrm{a}_{\mathrm{Ne}}\right)$. In addition, the pure detonation models (PD081, PD115) not only underproduce $\mathrm{Mn}$ with respect to Fe (like VM), but also eject a total amount of $\mathrm{Mn}$ roughly three orders of magnitude below all other explosions. Although we do not have solar counterparts of CSDD-L, CSDD-S, PD081, PD115 nor zerometallicity versions of $\mathrm{VM}$, the comparison between the individual CO detonations still indicates a metallicity-dependent Mn production.

In addition, some $\mathrm{Mn}$ is made via ${ }^{55} \mathrm{Fe}$ directly only in the $M_{\mathrm{Ch}}$ models. In Fig. 4 we can observe that ${ }^{55} \mathrm{Co}$ is not produced at the very tip of the high-density end of the tracer particle distribution in Model R60. These are exactly the conditions where the even more neutron rich element ${ }^{55} \mathrm{Fe}$ is synthesized. In contrast to $\mathrm{M} 2 \mathrm{a}_{\mathrm{Ne}}$, we do not observe any changes in the yields of R60 $0_{\mathrm{Ne}}$ compared to R60. This is most likely because almost all of ${ }^{55} \mathrm{Co}$ and ${ }^{55} \mathrm{Fe}$ is synthesized in normal freeze-out from NSE. Thus, the products largely depend on the neutronization due to electron capture during the explosion phase (see Sec. 2.2) and only weakly on the initial metallicity.

It should be noted that explosions such as M2a do not resemble normal SNe Ia in some aspects (see also Fink et al. 2010; Gronow et al. 2020). A solution to this problem might be to further decrease the mass of the helium shell (Townsley et al. 2019), but this also reduces the production of $\mathrm{Mn}$ in its detonation. However, explosions such as HeD-S are candidates for $\mathrm{Ca}$-rich transients, a sub-luminous class of $\mathrm{SNe}$ residing between normal SNe Ia and classical novae in terms of absolute magnitude. Frohmaier et al. (2018) estimate rather high rates for Ca-rich transients of about 33\%-94\% of the rate of normal $\mathrm{SNe}$ Ia. If this proves to be correct, such explosions may substantially contribute to the production of $\mathrm{Mn}$ in the Universe.

To estimate the effect of Ca-rich $\mathrm{SNe}$ on the evolution of $[\mathrm{Mn} / \mathrm{Fe}]$ in the Milky Way we carried out a chemical evolution simulation using the code One-zone Model for the Evolution of GAlaxies (OMEGA; Côté et al. 2017). We calculate an open-box model and employ the Côté et al. (2017) star formation model to control the in- and outflows of gas. These are linked to the star formation rate $M_{\star}$ via a mass loading factor $\eta$. The total mass of gas inside the Galaxy is determined by the star formation efficiency $f_{\star}: M_{\star}=f_{\star} M_{\text {gas }}$. The star formation rate is taken from Chiappini et al. (2001), yields for massive stars are from Limongi \& Chieffi (2018) (we use averaged values of their different rotating and non-rotating models), and yields of AGB stars are extracted from Karakas (2010). We find reasonable agreement with observational data for Mn using different SN Ia scenarios (see below) and fixing the star formation efficiency to $f_{\star}=0.006$, the mass loading factor to $\eta=0.7$, and the proportional constant connecting the star formation timescale and the dynamical timescale to $f_{\text {dyn }}=0.004$. Moreover, the mass

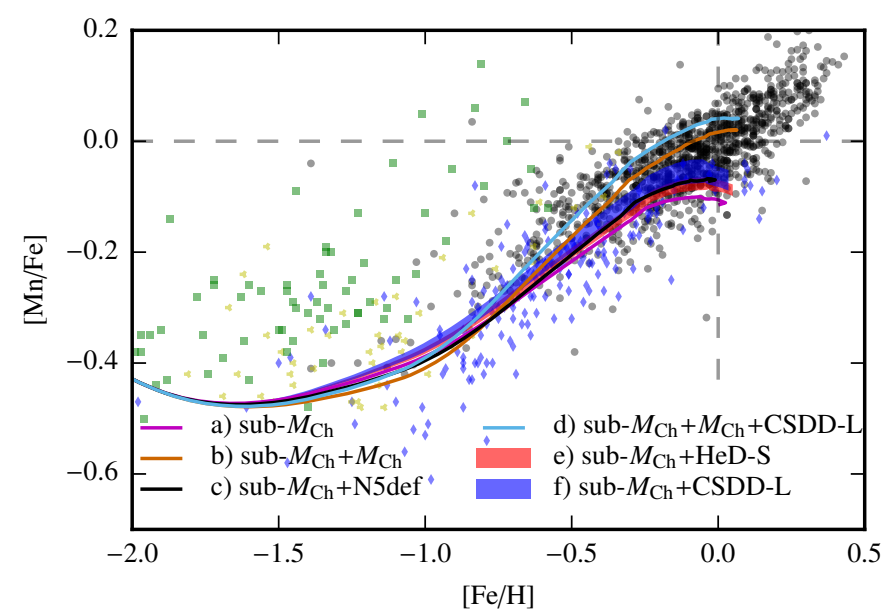

Fig. 5. Evolution of $[\mathrm{Mn} / \mathrm{Fe}]$ for different $\mathrm{SNe}$ Ia scenarios. The sub$M_{\mathrm{Ch}}$ scenario is represented by the Model VM, the $M_{\mathrm{Ch}}$ scenario by N100ddt, and N5def is used as a proxy for SNe Iax. The shaded areas in the runs sub- $M_{\mathrm{Ch}}+\mathrm{CSDD}-\mathrm{L}$ and sub- $M_{\mathrm{Ch}}+\mathrm{HeD}-\mathrm{S}$ correspond to a range in the rate of Ca-rich transients between $33 \%$ and $94 \%$. Black dots show observational data of Adibekyan et al. (2012), blue diamonds the data of Reddy et al. (2006), yellow triangles the data of Gratton et al. (2003), and red squares the data of Ishigaki et al. (2012, 2013). The data is from the STELLar ABundances (STELLAB) library (Ritter \& Côté 2016). The non-LTE data by Eitner et al. (2020) are not included in this simple approach.

transition between AGB yields and massive star yields is chosen to be $10.5 M_{\odot}$. Finally, the rate of SNe Ia is chosen to be $1.3 \times 10^{-3} M_{\odot}^{-1}$, and the total number of SNe Ia is distributed according to the chosen contribution of each channel (see cases below).

For the Chandrasekhar mass delayed detonations (N100ddt), helium-shell double-detonations (CSDD-L, HeD-S), and violent WD mergers (VM), we use delay time distributions calculated with the STARTRAcK binary evolution code (e.g., Belczynski et al. 2008; Ruiter et al. 2009). For this work we assume Chandrasekhar mass exploding CO WDs that have a hydrogen-rich donor (in most cases a subgiant or giant star) that produces delayed-detonations. For violent WD mergers, we include any merger between two CO WDs that has at least one component WD mass $\geq 0.9 M_{\odot}$. For double-detonations with helium shells we employ the WD mass-dependent helium shell prescription of Ruiter et al. (2014).

We implemented the following combinations of SN Ia channels. Combinations (a), (b), and (c) have also been investigated by Seitenzahl et al. (2013b):

(a) sub- $M_{\mathrm{Ch}}: 100 \%$ Model VM;

(b) sub- $M_{\mathrm{Ch}}+M_{\mathrm{Ch}}: 50 \%$ Model VM $+50 \%$ Model N100ddt;

(c) sub- $M_{\mathrm{Ch}}+$ Iax: $80 \%$ Model $\mathrm{VM}+20 \%$ N5def;

(d) sub- $M_{\mathrm{Ch}}+M_{\mathrm{Ch}}+$ CSDD-L: same as case (b) with $50 \%$ of CSDD-L added, which increases the number of SNe Ia per stellar mass formed to $1.95 \mathrm{M}_{\odot}^{-1}$;

(e) sub- $M_{\mathrm{Ch}}+\mathrm{HeD}-\mathrm{S}$ : the SN Ia rate consists of $100 \%$ of Model VM, and 33\%-94\% of Model HeD-S are included;

(f) sub- $M_{\mathrm{Ch}}+$ CSDD-L: same as case (e) with Model CSDD-L instead of HeD-S.

Combinations (a), (b), and (c) confirm the results described above. The solar value of $[\mathrm{Mn} / \mathrm{Fe}]$ can only be reached when including a significant fraction of $M_{\mathrm{Ch}}$ explosions. In addition, a rather high fraction of $20 \%$ of failed deflagrations (N5def) represented by case (c) is not sufficient to match the increasing 
trend. However, we note that the upward trend in $[\mathrm{Mn} / \mathrm{Fe}]$ is not only due to the contribution of $M_{\mathrm{Ch}}$-mass SNe Ia. CCSNe also exhibit an increasing trend in $[\mathrm{Mn} / \mathrm{Fe}]$ with increasing metallicity. This is illustrated in scenario (a) where $\mathrm{SNe}$ Ia have virtually no contribution to Mn. However, despite the CCSNcaused increase in $\mathrm{Mn}$, a solar ratio is not reached. Moreover, we find that helium detonations (HeD-S, CSDD-L) are as effective in increasing the final value of $[\mathrm{Mn} / \mathrm{Fe}]$ as N5def with our choice of rates (see variations (e) and (f)). Furthermore, case (d) demonstrates that CSDD-L also raises $[\mathrm{Mn} / \mathrm{Fe}]$ in the presence of N100ddt. This indicates that Ca-rich transients are able to reduce the need for $M_{\mathrm{Ch}}$ explosions to reproduce the evolution of $[\mathrm{Mn} / \mathrm{Fe}]$. We find that a reduction in $M_{\mathrm{Ch}}$ explosions to $30 \%$ of the SN Ia rate in scenario (d) yields $[\mathrm{Mn} / \mathrm{Fe}]$ values similar to those in case (b). Replacing CSDD-L with HeD-S allows a reduction to $40 \%$. Thus, the occurrence rate of $M_{\mathrm{Ch}} \mathrm{SNe}$ Ia needed to explain why $[\mathrm{Mn} / \mathrm{Fe}]$ versus $[\mathrm{Fe} / \mathrm{H}]$ can be reduced but not eliminated by the consideration of helium detonation models.

We note that this simple approach cannot replace future more elaborate GCE studies. It is only intended to give an impression of the contribution of helium shell detonation models compared to other SNe Ia scenarios given their low ejected mass per event. The delay-time distribution for explosions such as CSDD-L and HeD-S is not very well constrained, for instance. Fortunately, this does not challenge our conclusion since different DTDs only alter the shape of the $[\mathrm{Mn} / \mathrm{Fe}]$ evolution and leave the final value at $[\mathrm{Fe} / \mathrm{H}]=0$ largely unaffected. While there is still much uncertainty associated with the evolution and explosive outcome of helium shell double-detonation binaries, we note that in our binary evolution models progenitors of double-detonation SN Ia explosions are more similar in physical configuration to the models of Gronow et al. (2020) (in terms of core and shell mass), rather than the earlier models computed by Sim et al. (2012). However, it turns out that regardless of whether low to moderate shell mass systems (Gronow) or high shell mass systems (Sim) are actually contributing to the SN Ia population, it will not have any noticeable effect on the delay-time distribution of these explosions since the timescale on which these WDs accumulate helium is insignificant by comparison (on the order of $\sim 10 \mathrm{Myr}$ ).

In addition, a large number of helium detonations would lead to tensions in $[\mathrm{Ti} / \mathrm{Fe}]$ and $[\mathrm{V} / \mathrm{Fe}]$ since these elements are produced in super-solar amounts in them (see Fig. 3). This is yet another argument excluding helium shell detonations or double detonations as a replacement for $M_{\mathrm{Ch}} \mathrm{SNe}$ Ia models producing a solar $[\mathrm{Mn} / \mathrm{Fe}]$ value. As Ti and $\mathrm{V}$ are, however, underproduced over the whole metallicity range in current GCE studies (see Prantzos et al. 2018 and references therein), their site of production is not yet completely clarified. The yields derived from helium detonation models do not solve the problem. They imply an increased production at $[\mathrm{Fe} / \mathrm{H}] \approx 0$ and fail to provide a good fit to the $[\mathrm{Ti} / \mathrm{Fe}]$ and $[\mathrm{V} / \mathrm{Fe}]$ evolution at low metallicities. We find that a decrease in the CSDD-L rate to approximately $10 \%$ of the SN Ia rate resolves this tension and yields the solar value for $[\mathrm{Ti} / \mathrm{Fe}]$ and $[\mathrm{V} / \mathrm{Fe}]$ in scenario $\mathrm{d}$ ). However, this only allows for a reduction in the $M_{\mathrm{Ch}}$ events to $45 \%$ compared to $30 \%$ mentioned above.

\subsection{Zinc}

The element zinc ranges right beyond the iron peak and is of high interest for GCE since its origin has not been clarified yet. It has four stable isotopes, namely ${ }^{64} \mathrm{Zn},{ }^{66} \mathrm{Zn},{ }^{67} \mathrm{Zn}$, and ${ }^{68} \mathrm{Zn}$, of which ${ }^{64} \mathrm{Zn}$ is the most abundant in the solar neighborhood. The production mechanisms are therefore more diverse than in the case of Mn. Zinc abundances in the Galaxy have been measured already by Sneden et al. (1991) and later by Mishenina et al. (2002), Cayrel et al. (2004), and Nissen et al. (2007). All agree on its evolution: $\mathrm{Zn}$ exhibits high values of $[\mathrm{Zn} / \mathrm{Fe}] \approx 0.6$ at $[\mathrm{Fe} / \mathrm{H}] \approx-4$, which drop to solar at around $[\mathrm{Fe} / \mathrm{H}] \approx-2$. From there on they remain at $[\mathrm{Zn} / \mathrm{Fe}] \approx 0$. Some of the $\mathrm{Zn}$ abundance is synthesized during $\mathrm{He}$ or $\mathrm{C}$ burning via the s-process in massive stars, but the major contribution comes from explosive nucleosynthesis in supernovae. Standard CCSNe models (see, e.g., the yields of Woosley \& Weaver 1995) fall short in the production of $\mathrm{Zn}$. Only the introduction of hypernovae (HNe) can account for the solar value of [ $\mathrm{Zn} / \mathrm{Fe}]$ (Kobayashi et al. 2006), but the high values at very low metallicities are not reached within this model either.

The most commonly used SN Ia model for GCE calculations is the W7 model (Iwamoto et al. 1999): a 1D pure deflagration in a $M_{\mathrm{Ch}} \mathrm{CO}$ WD. This particular simulation yields only negligible amounts of $\mathrm{Zn}$ (see Table 2). Therefore, it has been a goal to explain the evolution of $\mathrm{Zn}$ with other production sites, although 1D double-detonation and pure helium detonation models calculated by Woosley \& Kasen (2011) show substantial amounts of $\mathrm{Zn}$. The recent work by Hirai et al. (2018) tries to explain the evolution of $\mathrm{Zn}$ with metallicity-dependent yields of CCSNe as well as HNe from Nomoto et al. (2013). They find that the inclusion of electron capture supernovae (ECSNe, yields from Wanajo et al. 2018) is necessary to match the high [Zn/Fe] values at low metallicity. Jones et al. (2019a) presented nucleosynthesis yields of thermonuclear ECSNe (tECSNe), i.e., the explosion of oxygen-neon WDs at densities around $10^{10} \mathrm{~g} \mathrm{~cm}^{-3}$. These models overproduce neutron-rich isotopes such as ${ }^{48} \mathrm{Ca}$, ${ }^{50} \mathrm{Ti}$, and ${ }^{54} \mathrm{Cr}$, as well as ${ }^{66} \mathrm{Zn}$ and other elements beyond the iron peak. In a follow-up study Jones et al. (2019b) showed that these models complement nicely the contribution of ECSNe to the chemical evolution of the Milky Way. Prantzos et al. (2018) cannot reproduce the evolution of $\mathrm{Zn}$ using the nucleosynthesis yields of rotating massive stars by Limongi \& Chieffi (2018).

In spite of this, a high contribution of $\mathrm{SNe}$ Ia to $\mathrm{Zn}$ was proposed by Matteucci et al. (1993) and later on by François et al. (2004). Mishenina et al. (2002) claim that SNe Ia are responsible for as much as $67 \%$ of the $\mathrm{Zn}$ production. Tsujimoto \& Nishimura (2018) investigate the evolution of $\mathrm{Zn}$ in the Galaxy using $\mathrm{Mg}$ instead of $\mathrm{Fe}$ as the reference element. Magnesium is an $\alpha$-element assumed to be solely produced in CCSNe, and thus it is more sensitive in detecting the contribution of sources other than CCSNe to a specific element. They discover a decreasing trend for $[\mathrm{Zn} / \mathrm{Mg}]$ for $[\mathrm{Zn} / \mathrm{Mg}] \lesssim-1$ and an increasing trend for higher metallicities. This increasing behavior coincides with the well-known kick-in of SNe Ia at $[\mathrm{Fe} / \mathrm{H}]=-1$. Consequently, they conclude that SNe Ia must be responsible for this behavior and suggest a scenario including a He detonation with strong $\alpha$-rich freeze-out. The decreasing trend at low metallicities is explained in their GCE model by the incorporation of magnetorotational $\mathrm{SNe}(\mathrm{MR} \mathrm{SNe})$, whose rate decreases with increasing metallicity.

No matter which combination of supernova scenarios accounts for the observed abundances at $[\mathrm{Fe} / \mathrm{H}] \lesssim-1$, it is very likely that $\mathrm{SNe}$ Ia also contribute to $\mathrm{Zn}$ in a non-negligible way, i.e., a significant underproduction would require an even larger contribution from CCSNe to keep $[\mathrm{Zn} / \mathrm{Fe}]$ near the solar value. The question is which scenario for SNe Ia synthesizes $[\mathrm{Zn} / \mathrm{Fe}]$ at around the solar ratio or higher and therefore contributes to the enrichment of galaxies with $\mathrm{Zn}$. 
Table 2. Total amount of stable $\mathrm{Zn}$ in $M_{\odot}$ and the radioactive and stable isotopes from which $\mathrm{Zn}$ originates (shown as percentages) at $100 \mathrm{~s}$.

\begin{tabular}{lccccccccccc}
\hline \hline Model & $\mathrm{Zn}$ & ${ }^{64} \mathrm{Zn}$ & ${ }^{66} \mathrm{Zn}$ & ${ }^{68} \mathrm{Zn}$ & ${ }^{64} \mathrm{Ga}$ & ${ }^{64} \mathrm{Ge}$ & ${ }^{66} \mathrm{Ge}$ & ${ }^{67} \mathrm{Ge}$ & ${ }^{68} \mathrm{Ge}$ & ${ }^{67} \mathrm{Cu}$ & ${ }^{66} \mathrm{Ni}$ \\
\hline PD081 & $3.49 \mathrm{e}-16$ & 97.9 & - & - & 1.5 & - & - & - & - & - & - \\
PD115 & $5.42 \mathrm{e}-05$ & 99.1 & - & - & - & - & - & - & - & - & - \\
VM & $2.21 \mathrm{e}-06$ & 1.6 & - & - & 9.4 & 28.3 & 60.6 & - & - & - & - \\
M2a $\odot$ & $1.71 \mathrm{e}-04$ & 4.2 & 10.3 & - & 7.8 & 39.1 & 34.0 & 1.2 & 2.1 & - & - \\
M2a & $1.35 \mathrm{e}-04$ & - & - & - & 11.1 & 58.4 & 29.1 & - & - & - & - \\
M2a & $4.44 \mathrm{e}-04$ & - & - & - & 13.0 & 79.9 & 5.4 & - & - & - & - \\
CSDD-L & $6.05 \mathrm{e}-06$ & 1.6 & - & - & 12.7 & 21.2 & 36.7 & 12.7 & 14.4 & - & - \\
CSDD-S & $7.49 \mathrm{e}-04$ & - & - & - & 7.0 & 85.4 & 6.9 & - & - & - & - \\
HeD-S & $7.41 \mathrm{e}-04$ & - & - & - & 6.8 & 85.5 & 7.0 & - & - & - & - \\
N5def & $6.58 \mathrm{e}-07$ & 8.5 & - & - & 6.0 & 28.5 & 56.2 & - & - & - & - \\
N100ddt & $3.12 \mathrm{e}-06$ & 5.6 & - & - & 5.9 & 30.9 & 57.2 & - & - & - & - \\
R60 & $1.80 \mathrm{e}-07$ & 4.7 & 2.9 & - & 4.1 & 33.6 & 53.0 & - & - & - & - \\
R60 & $1.92 \mathrm{e}-06$ & - & - & - & 3.9 & 31.6 & 49.6 & - & - & - & 14.1 \\
W7 & $4.93 \mathrm{e}-07$ & - & - & - & - & - & - & - & - & - & - \\
\hline
\end{tabular}

Notes. Only contributions larger than $1 \%$ are listed.

Table 3. Ratio of $\mathrm{Zn}$ to Fe compared to solar for the total explosion, to shell and core material only, and to stable zinc isotopes decayed at $2 \times 10^{9} \mathrm{yr}$.

\begin{tabular}{lccccccc}
\hline \hline Model & {$[\mathrm{Zn} / \mathrm{Fe}]$} & {$[\mathrm{Zn} / \mathrm{Fe}]_{\text {shell }}$} & {$[\mathrm{Zn} / \mathrm{Fe}]_{\text {core }}$} & ${ }^{64} \mathrm{Zn}$ & ${ }^{66} \mathrm{Zn}$ & ${ }^{67} \mathrm{Zn}$ & ${ }^{68} \mathrm{Zn}$ \\
\hline PD081 & -10.54 & - & - & $3.48 \mathrm{e}-16$ & - & - & - \\
PD115 & -1.31 & - & - & $5.38 \mathrm{e}-05$ & $3.57 \mathrm{e}-07$ & - & - \\
VM & -2.60 & - & - & $8.67 \mathrm{e}-07$ & $1.34 \mathrm{e}-06$ & - & - \\
M2a $\odot$ & -0.66 & 0.82 & -0.92 & $8.72 \mathrm{e}-05$ & $7.59-05$ & $2.48 \mathrm{e}-06$ & $5.11 \mathrm{e}-06$ \\
M2a & -0.77 & 0.77 & -1.09 & $9.46 \mathrm{e}-05$ & $3.92 \mathrm{e}-05$ & $5.60 \mathrm{e}-07$ & $3.45 \mathrm{e}-07$ \\
M2a & -0.25 & 1.02 & -0.48 & $4.16 \mathrm{e}-04$ & $2.46 \mathrm{e}-05$ & $1.52 \mathrm{e}-06$ & $1.64 \mathrm{e}-06$ \\
CSDD-L & -0.71 & 0.07 & -1.77 & $2.14 \mathrm{e}-06$ & $2.25 \mathrm{e}-06$ & $7.86 \mathrm{e}-07$ & $8.73 \mathrm{e}-07$ \\
CSDD-S & 0.40 & 0.87 & -1.44 & $6.94 \mathrm{e}-04$ & $5.19 \mathrm{e}-05$ & - & - \\
$\mathrm{HeD}-\mathrm{S}$ & 0.87 & 0.87 & - & $6.86 \mathrm{e}-04$ & $5.18 \mathrm{e}-05$ & - & - \\
N5def & -2.64 & - & - & $2.83 \mathrm{e}-07$ & $3.74 \mathrm{e}-07$ & - & - \\
N100ddt & -2.51 & - & - & $1.32 \mathrm{e}-06$ & $1.79 \mathrm{e}-06$ & - & - \\
R60 & -1.20 & - & - & $7.67 \mathrm{e}-07$ & $1.02 \mathrm{e}-06$ & $5.76 \mathrm{e}-09$ & $8.67 \mathrm{e}-09$ \\
R60 & -1.17 & - & - & $6.96 \mathrm{e}-07$ & $1.22 \mathrm{e}-06$ & $1.48 \mathrm{e}-09$ & $3.80 \mathrm{e}-10$ \\
W7 & -1.66 & - & - & - & - & - & - \\
\hline
\end{tabular}

Table 2 lists the total production of $\mathrm{Zn}$ in solar masses as well as the fraction of isotopes via which it is produced. In addition, Table 3 shows $[\mathrm{Zn} / \mathrm{Fe}]$ for the whole explosion and for the helium shell and core detonation separately in the case of a double detonation. It reveals that all models, except for those including a helium detonation (M2 $\mathrm{a}_{\odot}, \mathrm{M} 2 \mathrm{a}, \mathrm{CSDD}-\mathrm{S}, \mathrm{CSDD}-\mathrm{L}$, and HeD-S), severely underproduce $[\mathrm{Zn} / \mathrm{Fe}]$ compared to its solar value. While $M 2 \mathrm{a}_{\odot}, M 2 \mathrm{a}_{\mathrm{Ne}}, \mathrm{M} 2 \mathrm{a}$, and CSDD-L exhibit only a moderate underproduction, the remaining models synthesize super-solar ratios $[\mathrm{Zn} / \mathrm{Fe}]$. The $[\mathrm{Zn} / \mathrm{Fe}]$ value of the helium detonation is quite similar in M2 $\mathrm{a}_{\odot}, \mathrm{CSDD}-\mathrm{S}$, and HeD-S, but the result of the whole explosion is dominated by the core detonation for model $M 2 \mathrm{a}_{\odot}$. In contrast to the case of $\mathrm{Mn}$, the values of $[\mathrm{Zn} / \mathrm{Fe}]$ in $\mathrm{M} 2 \mathrm{a}$ are higher than its solar metallicity counterpart. The most important production channel is via the symmetric nucleus ${ }^{64} \mathrm{Ge}$, as is also the case for CSDD-S and HeD-S. In Model CSDD-L the $[\mathrm{Zn} / \mathrm{Fe}]$ yield of the helium shell detonation is significantly lower than in the other models. The reason for this is the lower density of the envelope (see argument (ii) in Sect. 3.1) because most $\mathrm{Zn}$ is produced above an initial density of $5.0 \times 10^{5} \mathrm{~g} \mathrm{~cm}^{-3}$. The lower density is also responsible for the difference in the contribution of ${ }^{64} \mathrm{Ge}$ compared to the other double detonations as it is produced at higher densities than ${ }^{66} \mathrm{Ge}$, for example.

Furthermore, it can be seen that the main production channels are ${ }^{64} \mathrm{Ga} \rightarrow{ }^{64} \mathrm{Zn}, \quad{ }^{64} \mathrm{Ge} \rightarrow{ }^{64} \mathrm{Ga} \rightarrow{ }^{64} \mathrm{Zn}$, and ${ }^{66} \mathrm{Ge} \rightarrow{ }^{66} \mathrm{Ga} \rightarrow{ }^{66} \mathrm{Zn}$ for the majority of models except for PD081 and PD115. These isotopes are produced either in $\alpha$-rich freeze-out from NSE or in the helium detonation (see Fig. 6).

Figure 6 also shows that $\mathrm{Zn}$ is primarily produced in the helium shell at relatively high densities. This region is not reached by CSDD-L, as discussed above. A direct production of $\mathrm{Zn}$ in the form of a high contribution from ${ }^{64} \mathrm{Zn}$ can only be observed in the pure detonations PD081 and PD115 and, to a much lesser extent, in the $M_{\mathrm{Ch}}$ models. However, the total amount of $\mathrm{Zn}$ falls short of that of Models CSDD-S, HeD-S, and $\mathrm{M} 2 \mathrm{a}_{\odot}$ by about two orders of magnitude. R60 and $\mathrm{M} 2 \mathrm{a}_{\odot}$ are the only models to directly produce a non-negligible amount of ${ }^{66} \mathrm{Zn}$. Moreover, the direct production of $\mathrm{Zn}$ isotopes $\left({ }^{64,66} \mathrm{Zn}\right)$ is clearly a metallicity effect. The corresponding simulations at lower metallicity shift the production of $\mathrm{Zn}$ to the symmetric isotope. This behavior is not observed for the other models at solar metallicity (N5def, N100ddt, VM). In contrast to our most recent simulations $\left(\mathrm{M} 2 \mathrm{a}_{\odot}\right.$ and $\mathrm{R} 60$, where the solar value for each 


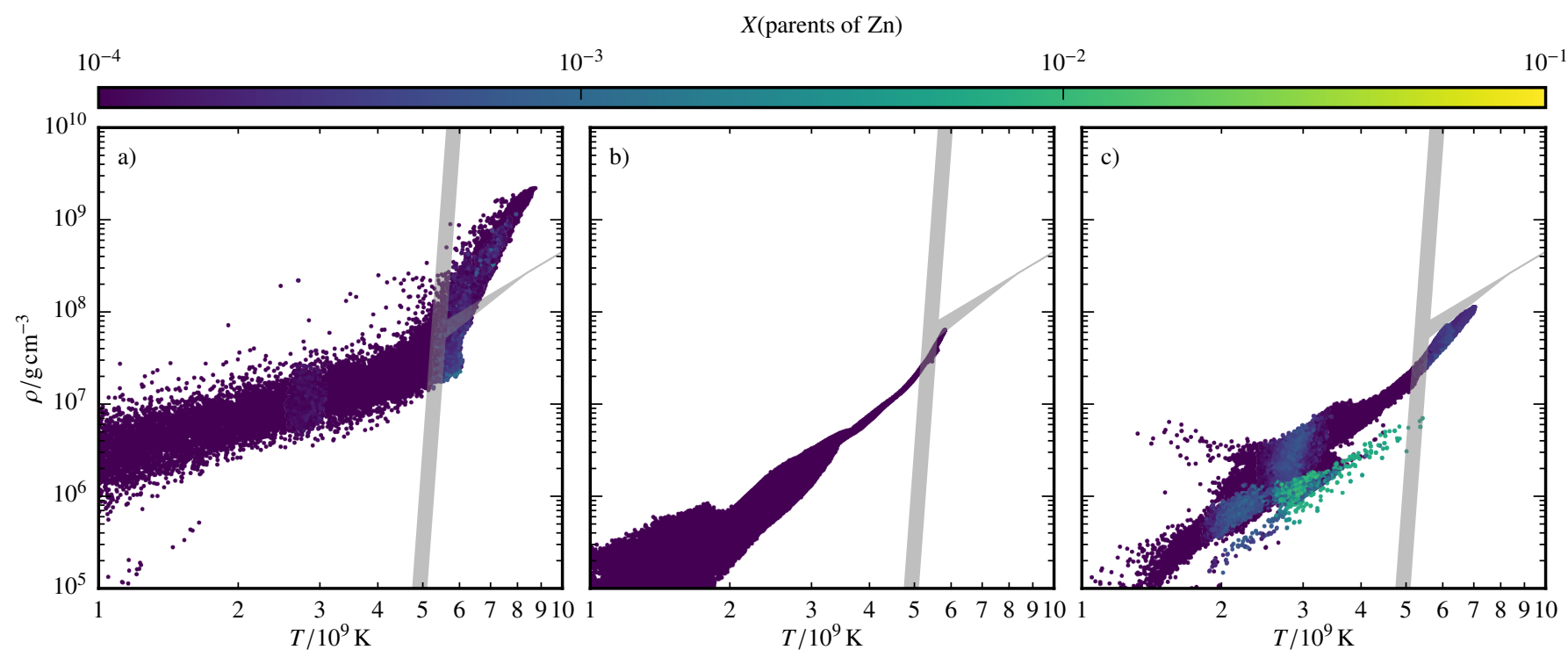

Fig. 6. Same as Fig. 2 with a color-coded mass fraction of the parent nuclei of Zn listed in Table 2. The panels show (a) R60, (b) VM, (c) M2a $\odot$.

isotope according to Asplund et al. 2009 is used as input for the postprocessing), the metallicity is set by adding only ${ }^{22} \mathrm{Ne}$ to adjust the electron fraction. Therefore, the lack of seed nuclei in the investigated region might affect the detailed nucleosynthesis results. This is confirmed by models $\mathrm{R} 60_{\mathrm{Ne}}$ and $\mathrm{M} 2 \mathrm{a}_{\mathrm{Ne}}$. They do not produce any $\mathrm{Zn}$ isotopes and also show differences in the production of various other species.

In summary, any SN Ia scenario noticeably contributing to the enrichment of the Galaxy with $\mathrm{Zn}$ is required to include a prominent helium detonation. The production of $\mathrm{Zn}$ can be explained by the same three arguments used for the case of $\mathrm{Mn}$. However, here CSDD-L shows the lowest ratio to iron since the initial shell density is too low to synthesize $\mathrm{Zn}$ in sufficient amounts. We abstain from adding an investigation of the galactic evolution of $[\mathrm{Zn} / \mathrm{Fe}]$ since we have already shown for the case of $\mathrm{Mn}$ that low-mass double detonations and pure helium shell detonations do contribute significantly to $[\mathrm{Mn} / \mathrm{Fe}]$ despite their low ejecta masses. This result holds analogously for $[\mathrm{Zn} / \mathrm{Fe}]$.

Instead, we briefly estimate the influence of ${ }^{68} \mathrm{Ge}$ on the light curve. All other isotopes listed in Table 2 are short-lived with a maximum half-life ${ }^{1}$ of $61.8 \mathrm{~h}$ for ${ }^{67} \mathrm{Cu}$. Although compared to ${ }^{56} \mathrm{Ni}$ long-lived isotopes are not produced in large amounts, they can modify the shape of the light curve at late times (Seitenzahl et al. 2009). Furthermore, $\gamma$ rays or X-rays emitted in their decay might be detectable.

${ }^{68} \mathrm{Ge}$ decays to ${ }^{68} \mathrm{Ga}$ via electron capture with a half-life of $T_{1 / 2}=270.95 \mathrm{~d}$. The two most relevant X-rays emitted have energies of $E_{1}=9.225$ and $E_{2}=9.252 \mathrm{keV}$ with an emission probability of $I_{1}=0.131$ and $I_{2}=0.258$, respectively. As an example, we consider Model M2 $\mathrm{a}_{\odot}$, which ejects $3.554 \times$ $10^{-6} M_{\odot}$ of ${ }^{68} \mathrm{Ge}$. Assuming transparent ejecta after $t=100 \mathrm{~d}$ and a very close $\mathrm{SN}$ explosion at a distance of $d=1 \mathrm{Mpc}$, we arrive at a flux on Earth of

$$
\begin{aligned}
F_{i} & =\frac{1}{4 \pi d^{2}} \cdot I \lambda N_{0} e^{-\lambda t} \cdot E_{i} \\
& = \begin{cases}2.325 \times 10^{-17} \mathrm{erg} \mathrm{s}^{-1} \mathrm{~cm}^{-2} & i=1 \\
4.592 \times 10^{-17} \mathrm{erg} \mathrm{s}^{-1} \mathrm{~cm}^{-2} & i=2,\end{cases}
\end{aligned}
$$

\footnotetext{
1 All nuclear decay data is taken from https://www.nndc.bnl. gov/nudat2/
}

where $\lambda$ is the decay constant and $N_{0}$ the number of nuclei at $t=0$, which can be derived dividing the ejected mass by the corresponding atomic mass ${ }^{2}$. The $\mathrm{X}$-ray telescope $N u S T A R$ (Harrison et al. 2013) has a sensitivity of $2 \times 10^{-15} \mathrm{erg} \mathrm{s}^{-1} \mathrm{~cm}^{-2}$ in the considered energy range. Therefore, the added flux of the two X-ray emissions is about a factor of 35 below the detection limit, and thus a higher production of ${ }^{68} \mathrm{Ge}$ would be necessary for a potential detection even under the rather favorable conditions assumed here. We note that this statement is by no means conclusive since the sensitivity of NUSTAR also depends on the line shape and on the observation time.

Subsequently, ${ }^{68} \mathrm{Ga}$ decays to ${ }^{68} \mathrm{Zn}$ via positron emission very quickly $\left(T_{1 / 2}=67.71 \mathrm{~min}\right)$. Assuming an instantaneous energy deposition, we calculated the contributions of ${ }^{68} \mathrm{Ga},{ }^{44} \mathrm{Sc}$, ${ }^{56} \mathrm{Co},{ }^{57} \mathrm{Co}$, and ${ }^{55} \mathrm{Fe}$ positrons, conversion electrons, and Auger electrons to the SN light curve for Models HeD-S (same amount of ${ }^{68} \mathrm{Ge}$ as CSDD-S), CSDD-L, and M2 $\mathrm{a}_{\odot}$. In the cases of ${ }^{68} \mathrm{Ga}$ and ${ }^{44} \mathrm{Sc}$ the electrons emitted by their long-lived parents are included as well. Figure 7 shows that with less than $0.4 \%$ of the energy generation per second $\epsilon$, the ${ }^{68} \mathrm{Ga}$ decay plays only a minor role in model $\mathrm{M} 2 \mathrm{a}_{\odot}$. Its contribution is even lower in the HeD-S and CSDD-L models.

\subsection{Copper}

Copper directly follows nickel in the periodic table and its elemental solar abundance consists of two stable isotopes, namely ${ }^{63} \mathrm{Cu}$ and ${ }^{65} \mathrm{Cu}$. Of these, about $69 \%$ are attributed to ${ }^{63} \mathrm{Cu}$ (Asplund et al. 2009). The first extensive measurements and an analysis of $\mathrm{Cu}$ abundances have been carried out by Sneden et al. (1991). The general trend of an increasing value of $[\mathrm{Cu} / \mathrm{Fe}]$ with $[\mathrm{Fe} / \mathrm{H}]$ in the Galaxy was established in that work. $[\mathrm{Cu} / \mathrm{Fe}]$ increases from a value of approximately -1 at $[\mathrm{Fe} / \mathrm{H}] \approx-3$ to the solar value at $[\mathrm{Fe} / \mathrm{H}] \approx 0$.

As for $\mathrm{Zn}$, the origin of $\mathrm{Cu}$ is still very uncertain. The largest contributors are believed to be the weak component of the s-process in massive stars (a secondary process) and the direct fusion as a primary element simultaneous to IGEs in CCSNe and SNe Ia. Matteucci et al. (1993) carried out detailed GCE

2 The atomic mass is taken from https://www-nds. iaea.org/ 


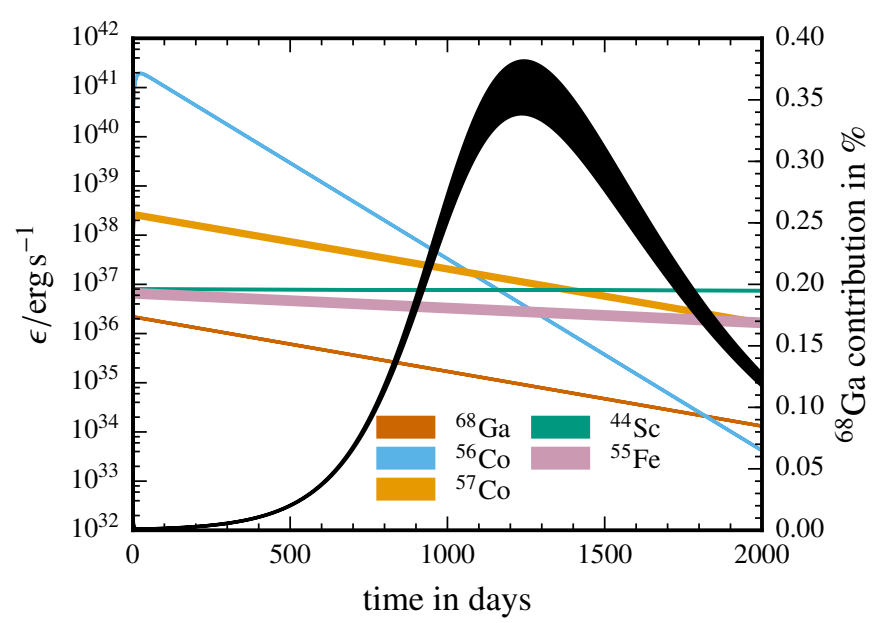

Fig. 7. Energy generation rate $\epsilon$ of the emission of positrons, conversion electrons, and Auger electrons in model $\mathrm{M} 2 \mathrm{a}_{\odot}$. Shaded areas show the range between no $\mathrm{X}$-ray trapping and full $\mathrm{X}$-ray trapping. The black curve depicts the contribution of the ${ }^{68} \mathrm{Ga}$ decay relative to the total energy generation.

calculations. Comparing their data to the Sneden et al. (1991) data, they conclude that a best fit is achieved if the SNe Ia yields for $\mathrm{Cu}$ are increased by an order of magnitude. Moreover, they claim that $\mathrm{SNe}$ Ia start contributing to the enrichment of the Galaxy starting from $[\mathrm{Fe} / \mathrm{H}] \approx-1.7$ (i.e., already in the halo phase). Mishenina et al. (2002) provide a large upgrade to the $\mathrm{Cu}$ abundance data by measuring $\mathrm{Cu}$ and $\mathrm{Zn}$ in 90 metalpoor stars in the Galaxy. They arrive at the conclusion that the increase in $[\mathrm{Cu} / \mathrm{Fe}]$ is due to a significant contribution of $\mathrm{SNe}$ Ia. As a guideline they also provide a very rough estimate of the relative contributions of $\mathrm{Cu}$ from different production sites to the solar abundance. They assign $7.5 \%$ to SNe II (primary process in massive stars), 25\% to secondary processes in massive stars, $5 \%$ to the s-process in AGB stars, and $62.5 \%$ to $\mathrm{SNe}$ Ia. The overall trend of the $\mathrm{Cu}$-to-Fe ratio, i.e., a sub-solar plateau at low metallicities and an increase to the solar value, which is reached at $[\mathrm{Fe} / \mathrm{H}] \approx-0.8$, has also been confirmed by Reddy et al. (2003). In contrast, Prantzos et al. (2018) show that by including the yields of Limongi \& Chieffi (2018) the evolution of $[\mathrm{Cu} / \mathrm{Fe}]$ can be modeled with $\mathrm{CCSNe}$ alone (they use W7 for SNe Ia). Nissen \& Schuster (2011) also confirm subsolar $[\mathrm{Cu} / \mathrm{Fe}]$ values for their low- $\alpha$ population of dwarf stars in the solar neighborhood. These stars are thought to be primarily enriched by SNe Ia, and therefore they conclude that they are not main contributors to $\mathrm{Cu}$. Simmerer et al. (2003) investigate the $\mathrm{Cu}$ abundances in various globular clusters and draw the opposite conclusion, that $\mathrm{SNe}$ Ia are likely to be the main contributors to $\mathrm{Cu}$, because $[\mathrm{Cu} / \mathrm{Fe}]$ in globular clusters follows the trend seen in field stars. However, the only cluster spanning a significant range in metallicity $(-1.8<[\mathrm{Fe} / \mathrm{H}]<0.8)$ is $\omega$-Centauri and there the $[\mathrm{Cu} / \mathrm{Fe}]$ curve is rather flat. This suggests a different chemical evolution history. In contrast to the previously mentioned results, Romano \& Matteucci (2007) find that their GCE model can fit both the Galaxy and $\omega$-Centauri if the s-process yields are enhanced and the SN Ia yields are reduced. Cunha et al. (2002) investigate in more detail the evolution of $\omega$-Centauri. They attribute the strong enhancement of $\alpha$-elements relative to $\mathrm{Fe}$ and the constant evolution of $[\mathrm{Cu} / \mathrm{Fe}]$ at a rather low value of -0.5 to an enrichment via CCSNe. However, a final explanation for the lack of SNe Ia enrichment is not given. Recent non-LTE $\mathrm{Cu}$ abundance measurements are presented by Yan et al. $(2015,2016)$. They show that non-LTE effects raise the previously measured values by about 0.2 dex and that this difference is also metallicity dependent. The impact of the non-LTE treatment is higher for lower $[\mathrm{Fe} / \mathrm{H}]$, and therefore flattens the whole curve. Furthermore, the plateau at lower metallicities is extended compared to older works, and reaches up to $[\mathrm{Fe} / \mathrm{H}] \approx-1$ and then rises to solar. However, Yan et al. (2015) note that due to the uncertainties in the main production site of $\mathrm{Cu}$ and to the peculiar evolution of $\mathrm{Cu}$ in Ursa Major, $\omega$-Centauri, the Sagittarius dwarf galaxy, and the halo subpopulation (Nissen \& Schuster 2011), further GCE models should be postponed until the trend of $[\mathrm{Cu} / \mathrm{Fe}]$ is clearly established by using non-LTE measurements.

Similar to the discussion on $\mathrm{Zn}$ (see Sect. 3.2), it is now interesting to see which SN Ia scenario is able to synthesize $\mathrm{Cu}$ in significant amounts. Table 4 displays the $\mathrm{Cu}$ yields together with the relative contribution of the most important production channels. In Table 5 the $[\mathrm{Cu} / \mathrm{Fe}]$ values and the remaining stable $\mathrm{Cu}$ isotopes can be found.

The overall result is the same as for the $\mathrm{Zn}$ case: $\mathrm{Cu}$ is largely underproduced compared to solar in all simulations except for two models including a He detonation, in this case HeD-S and CSDD-S. However, $[\mathrm{Cu} / \mathrm{Fe}]$ values are lower than $[\mathrm{Zn} / \mathrm{Fe}]$ for the helium shell, which also diminishes the total $[\mathrm{Cu} / \mathrm{Fe}]$ yields. $\mathrm{M} 2 \mathrm{a}_{\odot}, \mathrm{M} 2 \mathrm{a}_{\mathrm{Ne}}$, and M2a even approach the pure detonation PD115 due to the low ratio of shell mass to core mass (argument (i) in Sect. 3.1). A comparison between $\mathrm{M} 2 \mathrm{a}_{\odot}$ and $\mathrm{M} 2 \mathrm{a}$ shows that the metallicity has little impact on the total value of $[\mathrm{Cu} / \mathrm{Fe}]$. It only slightly changes the fractions of the parent nuclei that decay to stable $\mathrm{Cu}$. In particular, we find that the direct production of ${ }^{63} \mathrm{Cu}$ and ${ }^{65} \mathrm{Cu}$ is favored for higher metallicity and that the inclusion of only ${ }^{22} \mathrm{Ne}$ leads to a decrease in $[\mathrm{Cu} / \mathrm{Fe}]$ (see $\mathrm{R} 60_{\mathrm{Ne}}, \mathrm{M} 2 \mathrm{a}_{\mathrm{Ne}}$ ). Moreover, the difference in $[\mathrm{Cu} / \mathrm{Fe}]$ from the helium shell between CSDD-L and the other double detonations is less significant than for $[\mathrm{Zn} / \mathrm{Fe}] .{ }^{63} \mathrm{Ga}$, for example, is mainly produced at an initial density higher than the density at the base of the shell in CSDD-L, and therefore the relative contribution to stable $\mathrm{Cu}$ is shifted to ${ }^{65} \mathrm{Ga}$ and ${ }^{65} \mathrm{Ge}$ compared to Models CSDD-S and HeD-S.

In contrast to $\mathrm{Zn}$, a main production channel for $\mathrm{Cu}$ cannot be identified. While most models, except R60, produce some $\mathrm{Cu}$ via ${ }^{63} \mathrm{Zn}$, the helium detonations additionally show a major contribution from ${ }^{63} \mathrm{Ga},{ }^{65} \mathrm{Ga}$, and ${ }^{65} \mathrm{Ge}$. The $M_{\mathrm{Ch}}$ simulations and PD115 also synthesize considerable amounts of ${ }^{63} \mathrm{Cu}$, and $\mathrm{M} 2 \mathrm{a}_{\odot}$ exhibits contributions from almost every parent isotope listed in Table 4. This suggests that neither the core nor the helium detonation dominates the total $\mathrm{Cu}$ yields.

To sum up, the creation of a large amount of $\mathrm{Cu}$ in relation to $\mathrm{Fe}$ requires an even more prominent helium detonation than in the case of $\mathrm{Zn}$. We find in addition that a density at the base of the helium envelope exceeding that in Model CSDD-L of $5.92 \times 10^{5} \mathrm{~g} \mathrm{~cm}^{-3}$ is essential. From a GCE point of view the contribution of helium detonations to $\mathrm{Cu}$ is less significant than in the case of $\mathrm{Mn}$ or $\mathrm{Zn}$ among the investigated models. The only model expected to contribute considerably to $[\mathrm{Cu} / \mathrm{Fe}]$ is $\mathrm{HeD}-\mathrm{S}$. Also in this case we postpone detailed GCE modeling to future work.

Similar to the discussion of long-lived radioactive isotopes in Sect. 3.2 we take a brief look at ${ }^{65} \mathrm{Zn}$. The only other longlived isotope in Table 4 is ${ }^{63} \mathrm{Ni}$. However, it is only produced in very small amounts in model R60, and its half-life of $101.2 \mathrm{y}$ implies a very low rate of decay. ${ }^{65} \mathrm{Zn}$, in contrast, has a half-life of $T_{1 / 2}=243.93 \mathrm{~d}$ and emits an energetic $\gamma$ ray with an energy of $E=1115.539 \mathrm{keV}$ and an emission probability of $I=0.5004$. 
F. Lach et al.: Nucleosynthesis imprints from Type Ia supernovae

Table 4. Total amount of stable $\mathrm{Cu}$ in $M_{\odot}$ and the radioactive and stable isotopes from which $\mathrm{Cu}$ originates (shown as percentages) at $100 \mathrm{~s}$.

\begin{tabular}{lccccccccccc}
\hline \hline Model & $\mathrm{Cu}$ & ${ }^{63} \mathrm{Cu}$ & ${ }^{65} \mathrm{Cu}$ & ${ }^{63} \mathrm{Zn}$ & ${ }^{65} \mathrm{Zn}$ & ${ }^{63} \mathrm{Ga}$ & ${ }^{65} \mathrm{Ga}$ & ${ }^{65} \mathrm{Ge}$ & ${ }^{63} \mathrm{Ni}$ & ${ }^{65} \mathrm{Ni}$ & ${ }^{63} \mathrm{Co}$ \\
\hline PD081 & $1.66 \mathrm{e}-15$ & 33.4 & - & 66.0 & - & - & - & - & - & - & - \\
PD115 & $2.48 \mathrm{e}-05$ & 34.2 & - & 64.9 & - & - & - & - & - & - & - \\
VM & $3.37 \mathrm{e}-07$ & - & - & 74.2 & - & 8.8 & 13.9 & 2.0 & - & - & - \\
M2a $\odot$ & $2.43 \mathrm{e}-05$ & 10.6 & 10.8 & 15.5 & 3.6 & 10.5 & 33.4 & 12.6 & 2.2 & - & - \\
M2a & $1.60 \mathrm{e}-05$ & - & - & 20.2 & - & 16.9 & 45.1 & 17.3 & - & - & - \\
M2a & $3.03 \mathrm{e}-05$ & - & - & 18.2 & 3.0 & 23.5 & 41.9 & 14.1 & - & - & - \\
$\mathrm{CSDD}-\mathrm{L}$ & $3.44 \mathrm{e}-06$ & - & - & 27.0 & - & 16.0 & 26.2 & 30.4 & - & - & - \\
$\mathrm{CSDD}-\mathrm{S}$ & $1.29 \mathrm{e}-04$ & - & - & 18.8 & - & 57.7 & 9.9 & 13.6 & - & - & - \\
$\mathrm{H}$ D-S & $1.31 \mathrm{e}-04$ & - & - & 17.9 & - & 59.1 & 9.4 & 13.6 & - & - & - \\
N5def & $1.15 \mathrm{e}-07$ & 38.9 & 1.4 & 30.8 & - & 8.3 & 9.5 & 4.5 & 6.7 & - & - \\
N100ddt & $4.58 \mathrm{e}-07$ & 29.6 & - & 38.9 & - & 10.1 & 11.5 & 5.0 & 3.8 & - & - \\
R60 & $2.54 \mathrm{e}-07$ & 22.3 & 8.3 & 9.3 & 3.1 & 3.5 & 12.5 & 16.6 & 13.3 & 2.3 & 8.9 \\
R60 & $1.13 \mathrm{e}-07$ & 2.3 & - & 20.7 & - & 7.8 & 28.1 & 37.2 & - & - & 2.4 \\
W7 & $3.21 \mathrm{e}-07$ & - & - & - & - & - & - & & & & \\
\hline
\end{tabular}

Notes. Only contributions larger than $1 \%$ are listed.

Table 5. Ratio of $\mathrm{Cu}$ to Fe compared to solar for the total explosion, and for shell and core material only and stable copper isotopes decayed at $2 \times 10^{9} \mathrm{yr}$.

\begin{tabular}{lccccc}
\hline \hline Model & {$[\mathrm{Cu} / \mathrm{Fe}]$} & {$[\mathrm{Cu} / \mathrm{Fe}]_{\text {shell }}$} & {$[\mathrm{Cu} / \mathrm{Fe}]_{\text {core }}$} & ${ }^{63} \mathrm{Cu}$ & ${ }^{65} \mathrm{Cu}$ \\
\hline PD081 & -9.48 & - & - & $1.65 \mathrm{e}-15$ & $9.78 \mathrm{e}-18$ \\
PD115 & -1.25 & - & - & $2.55 \mathrm{e}-05$ & $2.33 \mathrm{e}-07$ \\
$\mathrm{VM}$ & -3.03 & - & - & $2.82 \mathrm{e}-08$ & $5.44 \mathrm{e}-08$ \\
$\mathrm{M} 2 \mathrm{a}_{\odot}$ & -1.13 & 0.43 & -1.48 & $9.62 \mathrm{e}-06$ & $1.47 \mathrm{e}-05$ \\
$\mathrm{M} 2 \mathrm{a}_{\mathrm{Ne}}$ & -1.31 & 0.39 & -1.93 & $5.95 \mathrm{e}-06$ & $1.01 \mathrm{e}-05$ \\
$\mathrm{M} 2 \mathrm{a}$ & -1.04 & 0.43 & -1.51 & $1.29 \mathrm{e}-05$ & $1.75 \mathrm{e}-05$ \\
$\mathrm{CSDD}-\mathrm{L}$ & -0.57 & 0.21 & -1.69 & $1.49 \mathrm{e}-06$ & $1.95 \mathrm{e}-06$ \\
$\mathrm{CSDD}-\mathrm{S}$ & 0.02 & 0.48 & -1.31 & $9.86 \mathrm{e}-05$ & $3.02 \mathrm{e}-05$ \\
$\mathrm{HeD}-\mathrm{S}$ & 0.50 & 0.50 & - & $1.01 \mathrm{e}-04$ & $3.01 \mathrm{e}-05$ \\
$\mathrm{~N} 5 \mathrm{def}$ & -3.01 & - & - & $9.76 \mathrm{e}-07$ & $1.77 \mathrm{e}-08$ \\
$\mathrm{~N} 100 \mathrm{ddt}$ & -2.95 & - & - & $3.78 \mathrm{e}-07$ & $8.04 \mathrm{e}-08$ \\
R60 & -1.67 & - & - & $1.45 \mathrm{e}-07$ & $1.09 \mathrm{e}-07$ \\
R60 & -2.02 & - & - & $3.84 \mathrm{e}-08$ & $7.50 \mathrm{e}-08$ \\
W7 & -1.46 & - & - & - & - \\
\hline
\end{tabular}

$X($ parents of $\mathrm{Cu})$

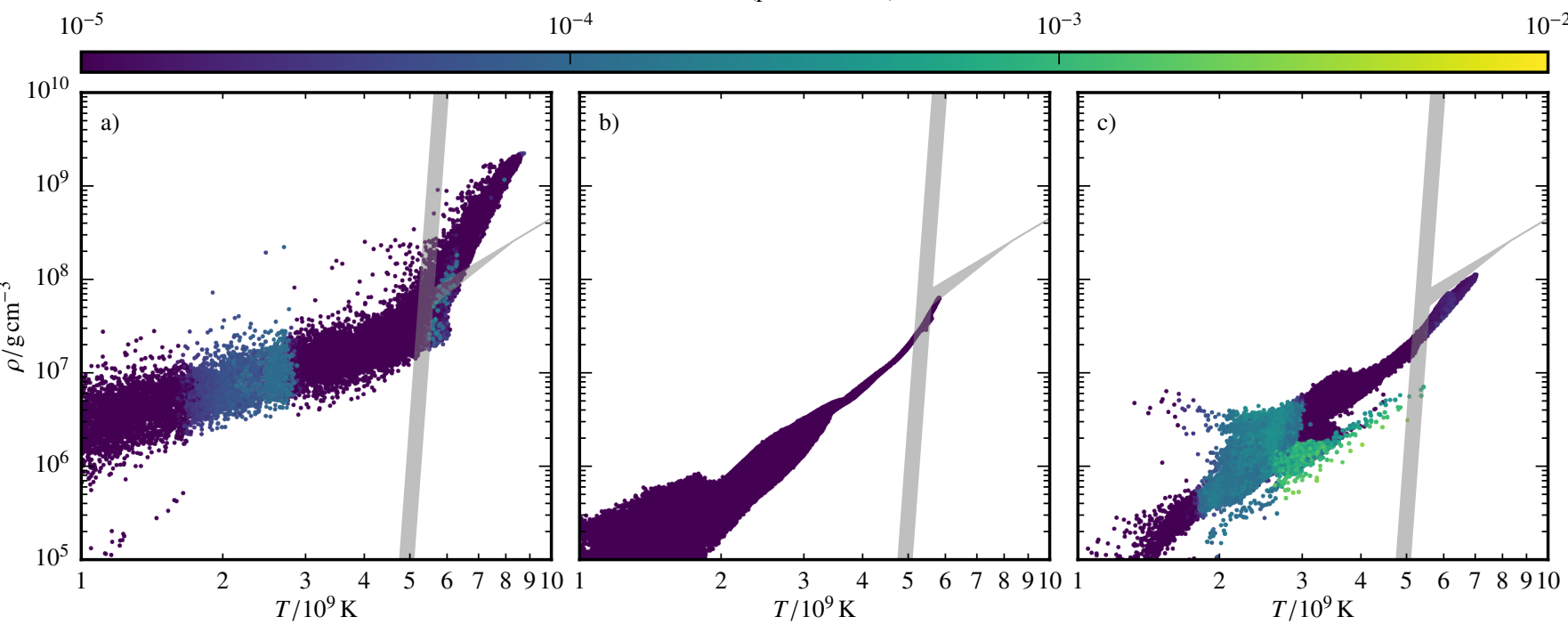

Fig. 8. Same as Fig. 2, with a color-coded mass fraction of ${ }^{64} \mathrm{Ge}$. The panels show (a) R60, (b) VM, (c) M2a $\mathrm{a}_{\odot}$. 
Most ${ }^{65} \mathrm{Zn}$ is synthesized via the fast decaying isotopes ${ }^{65} \mathrm{Ge}$ and ${ }^{65} \mathrm{Ga}$ (see Table 2). Under the same assumptions as in Sect. 3.2, we arrive at a flux on Earth for Model CSDD-S (highest amount of ${ }^{65} \mathrm{Zn}$ among our models with $3.019 \times 10^{-5} M_{\odot}$ ) of

$$
F=1.031 \times 10^{-13} \mathrm{erg} \mathrm{s}^{-1} \mathrm{~cm}^{-2} \text {. }
$$

Although this value is four orders magnitude higher than for the ${ }^{68} \mathrm{Ge}$ X-rays (see Sect. 3.2), it is still at least an order of magnitude below the expected sensitivity of the planned $\gamma$-ray telescope e-ASTROGAM (De Angelis et al. 2018), and therefore a detection is very unlikely.

\section{Summary}

We investigated the nucleosynthesis yields of a variety of SN Ia models from the HESMA (Kromer et al. 2017) archive as well as two new explosion simulations, $\mathrm{M} 2 \mathrm{a}_{\odot}$ and R60. These models include double-detonation models from Sim et al. (2012) and Gronow et al. (2020). Furthermore, we examined the pure detonations in sub- $M_{\mathrm{Ch}}$ WDs of Sim et al. (2010) and a set of $M_{\mathrm{Ch}}$ explosions from Seitenzahl et al. (2013a) and Fink et al. (2014).

Our aim was to identify elements characteristic of a certain explosion mechanism. In combination with GCE calculations and abundance measurements in stellar atmospheres, this can help to identify SN Ia progenitors. A well-known example is the element manganese. Its abundance relative to iron increases from $[\mathrm{Fe} / \mathrm{H}] \approx-1$ and it is not produced by CCSNe in sufficiently high ratios relative to $\mathrm{Fe}$. Therefore, it is attributed to thermonuclear explosions of $M_{\mathrm{Ch}}$ WDs (Seitenzahl et al. 2013b). In this study we find that super-solar amounts of Mn are additionally synthesized in helium detonations and that the actual result depends on the interplay of three parameters: the metallicity, the helium shell mass compared to the $\mathrm{CO}$ core, and the density of the helium envelope. A variation in these values gives rise to subsolar values of $[\mathrm{Mn} / \mathrm{Fe}]$ (as seen in Models M2a and CSDD-S) up to highly super-solar results (CSDD-L). This also brings the double-detonation scenario into play as a potential source of $\mathrm{Mn}$. Therefore, the sole distinction between sub- $M_{\mathrm{Ch}}$ and $M_{\mathrm{Ch}}$ mass models when investigating the source of $\mathrm{Mn}$ is insufficient. The details of the underlying model employed for the sub- $M_{\mathrm{Ch}}$ channel have to be defined in addition. Our GCE calculation corroborates this result and demonstrates that double-detonation models with massive helium shells can significantly reduce but not completely remove the need for $M_{\mathrm{Ch}}$ explosions to explain the solar $[\mathrm{Mn} / \mathrm{Fe}]$ ratio. Their actual rate is limited, however, due to their super-solar $[\mathrm{Ti} / \mathrm{Fe}]$ and $[\mathrm{V} / \mathrm{Fe}]$ values.

Moreover, the elements zinc and copper have received little attention when studying the contribution of SNe Ia to galactic chemical evolution, although Matteucci et al. (1993) already pointed out the potential relevance of these events as a production site. We find that double-detonation models are able to produce $\mathrm{Zn}$ and $\mathrm{Cu}$ in super-solar ratios with respect to Fe. Since a sophisticated GCE modeling and a comprehensive analysis of the contribution of massive stars is beyond the scope of this work, we did not include models for the evolution of $[\mathrm{Zn} / \mathrm{Fe}]$ and $[\mathrm{Cu} / \mathrm{Fe}]$. However, the general results from the case of Mn carry over to $\mathrm{Zn}$ and $\mathrm{Cu}$. The same three parameters as in the case of $\mathrm{Mn}$ also affect the creation of $\mathrm{Zn}$ and $\mathrm{Cu}$. Essentially, any value of $[\mathrm{Mn} / \mathrm{Fe}],[\mathrm{Zn} / \mathrm{Fe}]$, or $[\mathrm{Cu} / \mathrm{Fe}]$ can be realized using different combinations of helium shell mass, core mass, shell density and metallicity. However, in this study we find that the models from Sim et al. (2012) affect the evolution of $\mathrm{Mn}, \mathrm{Zn}$ and $\mathrm{Cu}$ the most. These models follow explosions in a system of a low-mass CO cores covered by a massive helium shell. They were intended to resemble a subluminous subclass of $\mathrm{SNe}$ Ia, namely calcium-rich transients, but do not account for normal SNe Ia. Their significance for GCE therefore depends on the realization frequency of Ca-rich transients, which is currently subject to large uncertainties. Ongoing and planned transient searches hold promise to clarify this aspect.

We thus emphasize that $\mathrm{SNe}$ Ia, or, in general thermonuclear explosions, should be treated more carefully in GCE studies. It seems necessary to include a variety of thermonuclear explosion models rather than sticking to only one or two scenarios. SNe Ia should be considered as a source of $\mathrm{Zn}$ and $\mathrm{Cu}$ in GCE simulations if the double-detonation scenario is used to represent either normal SNe Ia (see, however, Kromer et al. 2010, for potential problems with the predicted spectral observables) or the faint class of calcium-rich transients. $M_{\mathrm{Ch}}$ explosions might not be the only relevant source of $\mathrm{Mn}$.

Acknowledgements. This work was supported by the Deutsche Forschungsgemeinschaft (DFG, German Research Foundation) - Project-ID 138713538 SFB 881 ("The Milky Way System", subproject A10), by the ChETEC COST Action (CA16117), and by the National Science Foundation under Grant No. OISE-1927130 (IReNA). F. L. , F. K. R. and S. G. acknowledge support by the Klaus Tschira Foundation. IRS was supported by the Australian Research Council through Grant FT160100028. A. J. R. was supported by the Australian Research Council through Grant FT170100243. NumPy and SciPy (Oliphant 2007), IPython (Pérez \& Granger 2007), and Matplotlib (Hunter 2007) were used for data processing and plotting. The authors gratefully acknowledge the Gauss Centre for Supercomputing e.V. (www.gauss-centre.eu) for funding this project by providing computing time on the GCS Supercomputer JUWELS (Jülich Supercomputing Centre 2019) at Jülich Supercomputing Centre (JSC). Part of this research was undertaken with the assistance of resources and services from the National Computational Infrastructure (NCI), which is supported by the Australian Government, through the National Computational Merit Allocation Scheme and the UNSW HPC Resource Allocation Scheme. BC acknowledges support from the ERC Consolidator Grant (Hungary) funding scheme (project RADIOSTAR, G.A. n. 724560) and from the National Science Foundation (USA) under Grant No. PHY-1430152 (JINA Center for the Evolution of the Elements).

\section{References}

Adibekyan, V. Z., Sousa, S., Santos, N., et al. 2012, A\&A, 545, A32 Asplund, M., Grevesse, N., Sauval, A. J., \& Scott, P. 2009, ARA\&A, 47, 481 Bahcall, J. N. 1964, ApJ, 139, 318

Belczynski, K., Kalogera, V., Rasio, F. A., et al. 2008, ApJS, 174, 223

Bildsten, L., Shen, K. J., Weinberg, N. N., \& Nelemans, G. 2007, ApJ, 662, L95 Blinnikov, S. I., Röpke, F. K., Sorokina, E. I., et al. 2006, A\&A, 453, 229

Blondin, S., Kasen, D., Röpke, F. K., Kirshner, R. P., \& Mandel, K. S. 2011, MNRAS, 417, 1280

Brachwitz, F., Dean, D. J., Hix, W. R., et al. 2000, ApJ, 536, 934

Bravo, E. 2019, A\&A, 624, A139

Cayrel, R., Depagne, E., Spite, M., et al. 2004, A\&A, 416, 1117

Cescutti, G., \& Kobayashi, C. 2017, A\&A, 607, A23

Cescutti, G., Matteucci, F., Lanfranchi, G. A., \& McWilliam, A. 2008, A\&A, 491, 401

Chamulak, D. A., Brown, E. F., Timmes, F. X., \& Dupczak, K. 2008, ApJ, 677, 160

Chiappini, C., Matteucci, F., \& Romano, D. 2001, ApJ, 554, 1044

Côté, B., O’Shea, B. W., Ritter, C., Herwig, F., \& Venn, K. A. 2017, ApJ, 835, 128

Cunha, K., Smith, V. V., Suntzeff, N. B., et al. 2002, ApJ, 124, 379

De Angelis, A., Tatischeff, V., Grenier, I. A., et al. 2018, J. High Energy Astrophys., 19, 1

de los Reyes, M. A., Kirby, E. N., Seitenzahl, I. R., \& Shen, K. J. 2020, ApJ, 891,85

Eitner, P., Bergemann, M., Hansen, C. J., et al. 2020, A\&A, 635, A38

Fink, M., Hillebrandt, W., \& Röpke, F. K. 2007, A\&A, 476, 1133

Fink, M., Röpke, F. K., Hillebrandt, W., et al. 2010, A\&A, 514, A53

Fink, M., Kromer, M., Seitenzahl, I. R., et al. 2014, MNRAS, 438, 1762

Flörs, A., Spyromilio, J., Taubenberger, S., et al. 2020, MNRAS, 491, 2902

François, P., Matteucci, F., Cayrel, R., et al. 2004, A\&A, 421, 613

Frohmaier, C., Sullivan, M., Maguire, K., \& Nugent, P. 2018, ApJ, 858, 50 
F. Lach et al.: Nucleosynthesis imprints from Type Ia supernovae

Gratton, R. G., Carretta, E., Claudi, R., Lucatello, S., \& Barbieri, M. 2003, A\&A, 404, 187

Greggio, L., \& Renzini, A. 1983, A\&A, 118, 217

Gronow, S., Collins, C., Ohlmann, S. T., et al. 2020, A\&A, 635, A169

Harrison, F. A., Craig, W. W., Christensen, F. E., et al. 2013, ApJ, 770, 103

Hillebrandt, W., \& Niemeyer, J. C. 2000, ARA\&A, 38, 191

Hillebrandt, W., Kromer, M., Röpke, F. K., \& Ruiter, A. J. 2013, Front. Phys., 8, 116

Hirai, Y., Saitoh, T. R., Ishimaru, Y., \& Wanajo, S. 2018, ApJ, 855, 63

Hoyle, F., \& Fowler, W. A. 1960, ApJ, 132, 565

Hunter, J. D. 2007, Comput. Sci. Eng., 9, 90

Iben, I., Jr, \& Tutukov, A. V. 1984, ApJS, 54, 335

Inserra, C., Sim, S. A., Wyrzykowski, L., et al. 2015, ApJ, 799, L2

Ishigaki, M. N., Chiba, M., \& Aoki, W. 2012, ApJ, 753, 64

Ishigaki, M. N., Aoki, W., \& Chiba, M. 2013, ApJ, 771, 67

Iwamoto, K., Brachwitz, F., Nomoto, K., et al. 1999, ApJS, 125, 439

Jones, S., Côté, B., Röpke, F. K., \& Wanajo, S. 2019a, ApJ, 882, 170

Jones, S., Röpke, F., Fryer, C., et al. 2019b, A\&A, 622, A74

Jülich Supercomputing Centre 2019, J. Large-scale Res. Facil., 5,

Karakas, A. I. 2010, MNRAS, 403, 1413

Kasen, D., \& Plewa, T. 2007, ApJ, 662, 459

Kashi, A., \& Soker, N. 2011, MNRAS, 417, 1466

Khokhlov, A. M. 1984, Sov. Astron. Lett., 10, 123

Khokhlov, A. M., \& Érgma, É. V. 1985, Astrofizika, 23, 605

Kobayashi, C., \& Nomoto, K. 2009, ApJ, 707, 1466

Kobayashi, C., Umeda, H., Nomoto, K., Tominaga, N., \& Ohkubo, T. 2006, ApJ, 653,1145

Kobayashi, C., Karakas, A. I., \& Umeda, H. 2011, MNRAS, 414, 3231

Kobayashi, C., Nomoto, K., \& Hachisu, I. 2015, ApJ, 804, L24

Kobayashi, C., Leung, S.-C., \& Nomoto, K. 2020, ApJ, 895, 138

Kromer, M., Sim, S. A., Fink, M., et al. 2010, ApJ, 719, 1067

Kromer, M., Pakmor, R., Taubenberger, S., et al. 2013, ApJ, 778, L18

Kromer, M., Ohlmann, S. T., Pakmor, R., et al. 2015, MNRAS, 450, 3045

Kromer, M., Ohlmann, S., \& Röpke, F. K. 2017, Mem. Soc. Astron. It., 88, 312

Langanke, K., \& Martinez-Pinedo, G. 2000, Nucl. Phys. A, 673, 481

Limongi, M., \& Chieffi, A. 2018, ApJS, 237, 13

Marquardt, K. S., Sim, S. A., Ruiter, A. J., et al. 2015, A\&A, 580, A118

Matteucci, F., \& Greggio, L. 1986, A\&A, 154, 279

Matteucci, F., \& Recchi, S. 2001, ApJ, 558, 351

Matteucci, F., Raiteri, C., Busson, M., Gallino, R., \& Gratton, R. 1993, A\&A, 272, 421

Matteucci, F., Panagia, N., Pipino, A., et al. 2006, MNRAS, 372, 265

McWilliam, A. 1997, ARA\&A, 35, 503

McWilliam, A., Piro, A. L., Badenes, C., \& Bravo, E. 2018, ApJ, 857, 97

Meakin, C. A., Seitenzahl, I., Townsley, D., et al. 2009, ApJ, 693, 1188

Mishenina, T., Kovtyukh, V., Soubiran, C., Travaglio, C., \& Busso, M. 2002, A\&A, 396, 189

Nissen, P. E., \& Schuster, W. J. 2011, A\&A, 530, A15

Nissen, P. E., Akerman, C., Asplund, M., et al. 2007, A\&A, 469, 319

Nomoto, K., Kobayashi, C., \& Tominaga, N. 2013, ARA\&A, 51, 457

North, P., Cescutti, G., Jablonka, P., et al. 2012, A\&A, 541, A45

Oliphant, T. E. 2007, Comput. Sci. Eng., 9, 10

Pakmor, R., Edelmann, P., Röpke, F. K., \& Hillebrandt, W. 2012a, MNRAS, 424, 2222

Pakmor, R., Kromer, M., Taubenberger, S., et al. 2012b, ApJ, 747, L10

Pérez, F., \& Granger, B. E. 2007, Comput. Sci. Eng., 9, 21

Perlmutter, S., Aldering, G., Goldhaber, G., et al. 1999, ApJ, 517, 565

Phillips, M. M. 1993, ApJ, 413, L105

Piro, A. L., \& Bildsten, L. 2008, ApJ, 673, 1009
Prantzos, N., Abia, C., Limongi, M., Chieffi, A., \& Cristallo, S. 2018, MNRAS, 476, 3432

Rauscher, T., \& Thielemann, F.-K. 2000, At. Data Nucl. Data Tables, 75, 1

Reddy, B. E., Tomkin, J., Lambert, D. L., \& Allende Prieto, C. 2003, MNRAS, 340,304

Reddy, B. E., Lambert, D. L., \& Prieto, C. A. 2006, MNRAS, 367, 1329

Reinecke, M., Hillebrandt, W., \& Niemeyer, J. C. 1999, A\&A, 347, 739

Reinecke, M., Hillebrandt, W., \& Niemeyer, J. C. 2002, A\&A, 386, 936

Riess, A. G., Filippenko, A. V., Challis, P., et al. 1998, AJ, 116, 1009

Ritter, C., \& Côté, B. 2016, NuPyCEE: NuGrid Python Chemical Evolution Environment (Astrophysics Source Code Library)

Romano, D., \& Matteucci, F. 2007, MNRAS, 378, L59

Röpke, F. K. 2005, A\&A, 432, 969

Röpke, F. K. 2017, in Handbook of Supernovae, eds. A. Alsabti, \& P. Murdin (Springer)

Röpke, F. K., Kromer, M., Seitenzahl, I. R., et al. 2012, ApJ, 750, L19

Ruiter, A. J., Belczynski, K., \& Fryer, C. 2009, ApJ, 699, 2026

Ruiter, A. J., Belczynski, K., Sim, S. A., Seitenzahl, I. R., \& Kwiatkowski, D. 2014, MNRAS, 440, L101

Seitenzahl, I. R., \& Townsley, D. M. 2017, in Nucleosynthesis in Thermonuclear Supernovae, eds. A. W. Alsabti, \& P. Murdin (Springer), 1955

Seitenzahl, I. R., Taubenberger, S., \& Sim, S. A. 2009, MNRAS, 400, 531

Seitenzahl, I. R., Cescutti, G., Röpke, F. K., Ruiter, A. J., \& Pakmor, R. 2013a, A\&A, 559, L5

Seitenzahl, I. R., Ciaraldi-Schoolmann, F., Röpke, F. K., et al. 2013b, MNRAS, 429, 1156

Shen, K. J., \& Bildsten, L. 2009, ApJ, 699, 1365

Shen, K. J., \& Moore, K. 2014, ApJ, 797, 46

Shen, K. J., Kasen, D., Weinberg, N. N., Bildsten, L., \& Scannapieco, E. 2010, ApJ, 715, 767

Sim, S. A., Röpke, F. K., Hillebrandt, W., et al. 2010, ApJ, 714, L52

Sim, S. A., Fink, M., Kromer, M., et al. 2012, MNRAS, 420, 3003

Sim, S. A., Seitenzahl, I. R., Kromer, M., et al. 2013, MNRAS, 436, 333

Simmerer, J., Sneden, C., Ivans, I. I., et al. 2003, ApJ, 125, 2018

Sneden, C., Gratton, R., \& Crocker, D. 1991, A\&A, 246, 354

Springel, V. 2010, MNRAS, 401, 791

Taubenberger, S. 2017, Handbook of Supernovae (Springer), 317

Thielemann, F.-K., Nomoto, K., \& Yokoi, K. 1986, A\&A, 158, 17

Timmes, F. X., \& Arnett, D. 1999, ApJS, 125, 277

Timmes, F. X., Woosley, S. E., \& Weaver, T. A. 1995, ApJS, 98, 617

Timmes, F. X., Brown, E. F., \& Truran, J. W. 2003, ApJ, 590, L83

Tinsley, B. 1979, ApJ, 229, 1046

Townsley, D. M., Miles, B. J., Shen, K. J., \& Kasen, D. 2019, ApJ, 878, L38

Travaglio, C., Hillebrandt, W., Reinecke, M., \& Thielemann, F.-K. 2004, A\&A, 425, 1029

Travaglio, C., Gallino, R., Rauscher, T., et al. 2014, ApJ, 795, 141

Tsujimoto, T., \& Nishimura, N. 2018, ApJ, 863, L27

Wanajo, S., Müller, B., Janka, H.-T., \& Heger, A. 2018, ApJ, 852, 40

Wang, B., \& Han, Z. 2012, New Astron. Rev., 56, 122

Weinberg, D. H., Holtzman, J. A., Hasselquist, S., et al. 2019, ApJ, 874, 102

Whelan, J., \& Iben, I. J. 1973, ApJ, 186, 1007

Willcox, D. E., Townsley, D. M., Calder, A. C., Denissenkov, P. A., \& Herwig, F. 2016, ApJ, 832, 13

Woosley, S. E., \& Kasen, D. 2011, ApJ, 734, 38

Woosley, S. E., \& Weaver, T. A. 1995, ApJS, 101, 181

Woosley, S. E., Arnett, W. D., \& Clayton, D. D. 1973, ApJS, 26, 231

Yamaguchi, H., Badenes, C., Foster, A. R., et al. 2015, ApJ, 801, L31

Yan, H., Shi, J., \& Zhao, G. 2015, ApJ, 802, 36

Yan, H., Shi, J., Nissen, P. E., \& Zhao, G. 2016, A\&A, 585, A102 